QMW-PH-98-30

hep-th/9807127

July 1998

\title{
Duality and the Signature of Space-Time
}

\author{
C.M. HulL \\ Physics Department, Queen Mary and Westfield College, \\ Mile End Road, London E1 4NS, U.K.
}

\begin{abstract}
Versions of M-theory are found in spacetime signatures $(9,2)$ and $(6,5)$, in addition to the usual M-theory in $10+1$ dimensions, and these give rise to type IIA string theories in 10-dimensional spacetime signatures $(10,0),(9,1),(8,2),(6,4)$ and $(5,5)$, and to type IIB string theories in signatures $(9,1),(7,3)$ and $(5,5)$. The field theory limits are 10 and 11 dimensional supergravities in these signatures. These theories are all linked by duality transformations which can change the number of time dimensions as well as the number of space dimensions, so that each should be a different limit of the same underlying theory.
\end{abstract}




\section{Introduction}

In recent years, it has been discovered that dualities can relate theories with different gauge groups, different space-time dimensions, different amounts of supersymmetry, and even relate theories of gravity to gauge theories. Thus many of the concepts that had been thought absolute are now understood as relative: they depend on the 'frame of reference' used, where the concept of frame of reference is generalised to include the values of the various coupling constants. For example, the description of a given system when a certain coupling is weak can be very different from the description at strong coupling, and the two regimes can have different spacetime dimension, for example. However, in all this, one thing that has remained unchanged is the number of time dimensions; all the theories considered are formulated in a Lorentzian signature with one time coordinate, although the number of spatial dimensions can change. In this paper, it will be argued that

dualities can change the number of time dimensions as well, giving rise to exotic spacetime signatures.

The strong coupling limit of the type IIA superstring is a theory in $10+1$ dimensions whose low energy limit is 11-dimensional supergravity theory and which is referred to as M-theory. The type I, type II and heterotic superstring theories and certain supersymmetric gauge theories emerge as different limits of M-theory. The M-theory in $10+1$ dimensions will be linked via dualities to a theory in $9+2$ dimensions (9 space and 2 time dimensions), which will be referred to as $M^{*}$ theory, and a theory in $6+5$ dimensions, $M^{\prime}$-theory. Various limits of these will give rise to IIA-like string theories in $10+0,9+1,8+2,6+4$ and $5+5$ dimensions, and to IIB-like string theories in $9+1,7+3$, and $5+5$ dimensions. The field theory limits are supergravity theories with 32 supersymmetries in 10 and 11 dimensions with these signatures, many of which are new. Further dualities relate these to supersymmetric gauge theories in various signatures and dimensions, such as $2+2,3+1$ and $4+0$.

The resulting picture is that there should be some underlying fundamental 
theory and that different spacetime signatures as well as different dimensions can arise in various limits. The new theories are different real forms of the complexification of the original M-theory and type II string theories, perhaps suggesting an underlying complex nature of spacetime.

Each of the theories has a flat-space solution $\mathbb{R}^{p, q}$ of the appropriate signature, but they are linked via compactifications on tori $T^{m, n}$ with $m$ compact space dimensions and $n$ compact time dimensions. The starting point is to note that conventional string theory and M-theory with Lorentzian signature have classical solutions which are flat spaces of the form $\mathbb{R}^{m} \times T^{n, 1}$ where $T^{n, 1}$ is a Lorentzian torus with $n$ spacelike circles and one timelike circle. The presence of closed timelike loops means that the physics in such spaces is unusual, but it has often been fruitful in the past to study solutions that have little in common with the real world. An important issue with these solutions (as with many others) is whether a consistent quantum theory can be formulated in such backgrounds. Wave equations or Schrödinger equations can be solved with periodic time, but issues of measurement and collapse of the wave-function are problematic. In string theory, it is straightforward to study the solutions of the physical state conditions, but there are new issues that arise from strings (and branes) winding around the compact time.

Assuming that such timelike compactifications are consistent, then it is of interest to investigate the 'strong coupling' limits that arise in going to the boundaries of the moduli space and, surprisingly, the resulting limits give unexpected new theories. A superstring theory in $9+1$ dimensions compactified on a timelike circle gives, in the limit in which the circle shrinks to zero size, another superstring theory in $9+1$ dimensions related by timelike T-duality. Timelike T-duality takes the heterotic string back to the heterotic string [1], but it was shown in [2] that the timelike T-duals of the type IIA or type IIB strings are 'new' theories, the type $I I B^{*}$ or type $I I A^{*}$ strings, respectively. The strong coupling limit of the $I I A^{*}$ theory is the $M^{*}$-theory in $9+2$ dimensions, as will be shown in section 6 . Whereas M-theory compactified on a Euclidean 3-torus $T^{3,0}$ gives M-theory again in the limit in which the torus shrinks to zero size, M-theory compactified on a 
Lorentzian 3-torus $T^{2,1}$, in the limit in which the torus shrinks to zero size, gives the $M^{*}$ theory in $9+2$ dimensions. Next compactifying $M^{*}$ theory on a Euclidean 3 -torus $T^{3,0}$ gives, in the zero size limit, the $M^{\prime}$ theory in $6+5$ dimensions. Compactifying the $M, M^{*}, M^{\prime}$ theories on circles or 2-tori of various signatures and taking the zero volume limit then give rise to the various 10-dimensional string theories. These 11 and 10-dimensional theories have brane solutions with various world-volume signatures $[2,3]$ and some of these interpolate between flat space and solutions which are the product of a symmetric space $S O(p, q) / S O(p, q-1)$, which is a generalised de Sitter or anti de Sitter space of signature $(p, q-1)$, with an internal hyperbolic or spherical symmetric space. The world-volume theory is a superconformal gauge theory in a spacetime with signature $(p-1, q-1)$ which is invariant under the conformal group $S O(p, q)$. This leads to a duality between the superconformal gauge theory and the string or M-theory in the de Sitter-type space [2], by arguments similar to those in [4].

It has been known for some time that there are supersymmetric theories in non-Lorentzian signatures, such as $2+2$ or $5+5$ [5], and branes of various signatures were discussed in [6]. The considerations here lead to new supergravity theories to add to this list, and the string or M-theories that lead to these are all related to one another and to the supersymmetric gauge theories in various signatures by dualities, thereby unifying the many maximally supersymmetric theories in diverse signatures. The web of dualities linking these theories has a robust structure which survives many consistency checks, and it is remarkable that the theories that arise are all consistent with supersymmetry; for example, IIB theories arise in precisely those signatures admitting chiral fermions and real self-dual 5-forms.

Many of the theories that arise in this way appear to have pathologies, such as ghosts, tachyons or instabilities. Most of the theories have instantons $[2,3,7,8]$ that may lead to the instability of flat space, but, at least in some cases, there is an alternative interpretation of these solutions [2] and the question of vacuum decay is unclear. 
To summarise, M-theory and superstring theory have classical solutions that have periodic time. If a consistent quantum string or M-theory exists in such backgrounds, then dualities lead to the new theories with exotic signatures that are the subject of this paper, and the fact that the new theories are the usual M-theory or string theory written in terms of dual variables would mean that they are no worse than the original theory (with compact time) and some of the features that appear to be pathological are in fact the consequence of using unusual variables to describe the theory; this will be discussed further in section 3. It would of course be important and interesting to understand how physics might work in such theories directly, without recourse to duality arguments. These theories in signature $(s, t)$ also have decompactification limits which are flat solutions $\mathbb{R}^{s, t}$, and the question arises as to whether the theories in these backgrounds are consistent or have some pathology. For example, although the various 'wrong' signs occuring in the theories suggest instabilities, in some cases at least some of the apparent instabilities appear to be absent [2]. Of course, it may be that M-theory or string theory with periodic time is in fact pathological and then the new theories related to these by duality would also be pathological, and these pathologies would have been introduced by introducing closed timelike curves. However, the new theories can be written down in flat space without any reference to timelike curves, and the timelike compactifications are used only to relate the theories one to another. (It is perhaps worth mentioning that string theory in backgrounds in which time as well as all the space dimensions are compact have played an important role in the study of vertex algebras, the monster group and related areas of mathematics; see e.g. [1,9].) It is an important feature that all the exotic new theories are linked to the usual M-theory by chains of dualities, so that there should still be a single underlying theory. However, it seems that M-theory, or whatever the underlying theory is to be called, may have 'phases' in which the physics is more unusual than previously suspected. 


\section{The Type $I I^{*}$ Superstring Theories}

T-duality on a space-like circle interchanges the IIA and IIB string theories $[10,11]$, but for a timelike circle, T-duality does not take the IIA string theory to a IIB string theory or the IIB to IIA [13,2]. In [2], the images of the IIA and IIB theories under timelike T-duality, the $I I B^{*}$ and $I I A^{*}$ theories respectively, were investigated. The $I I A^{*}$ and $I I B^{*}$ theories can be obtained from the IIA and IIB theories, respectively, by acting with $i^{F_{L}}$ where $F_{L}$ is the left-handed fermion number. The zero-slope limits give corresponding supergravity actions, which differ from the usual IIA and IIB supergravities by certain signs; in particular, the signs of the kinetic terms of the RR gauge fields are all reversed, and this leads to the presence of E-brane solutions instead of the D-branes of the type II theories. An En-brane arises from imposing Dirichlet boundary conditions in time as well as in $9-n$ spatial coordinates, and is associated with an extended object with $n$ spacelike dimensions occurring at a particular instant in time. The En-branes of the type $I I^{*}$ theories are related to the $\mathrm{D} n$-branes of the type II theories by a timelike T-duality. The T-dualities linking the type II and type $I I^{*}$ theories are illustrated in the following diagram.

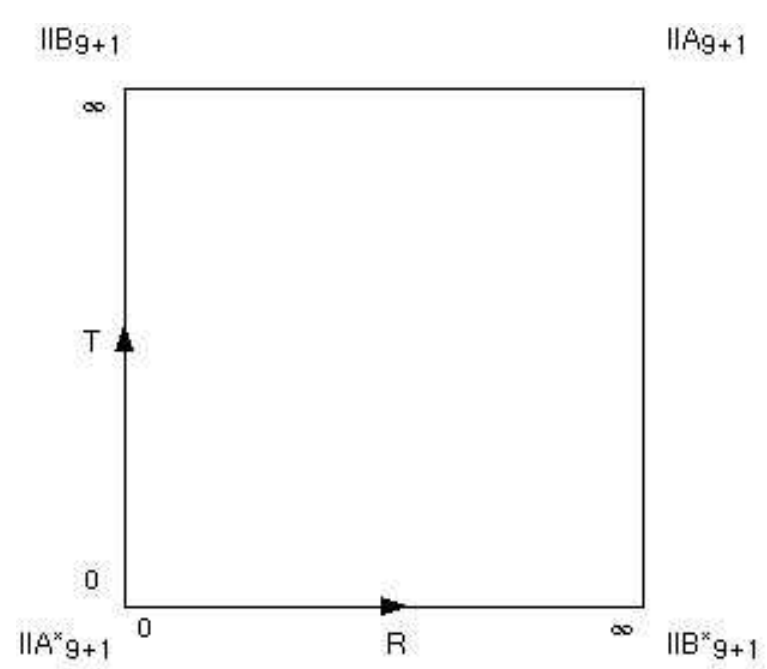

Figure 1 The moduli space for the type IIA string theory compactified on a Lorentzian torus $T^{1,1}$ with spacelike radius $R$ and timelike radius $T$. 
A spacelike T-duality relates the IIA with the IIB theory and the $I I A^{*}$ with the $I I B^{*}$ theory, while a timelike T-duality links the $I I A$ with the $I I B^{*}$ theory and the $I I B$ with the $I I A^{*}$ theory.

The type $I I^{*}$ theories have a twisted $N=2$ superalgebra

$$
\left\{Q_{i}, Q_{j}\right\}=\eta_{i j}\left(\Gamma^{\mu} C^{-1}\right) P_{\mu}
$$

where $i, j=1,2$ labels the two supercharges (which have the same chirality in the $I I B^{*}$ theory and opposite chirality in the $I I A^{*}$ theory), $C$ is the charge conjugation matrix and $\eta_{i j}$ is the $S O(1,1)$ invariant metric $\operatorname{diag}(1,-1)$. The anti-commutator of the second supercharge with itself has the 'wrong' sign. The scalars of the II B* theory take values in the coset space

$$
\frac{S L(2, \mathbb{R})}{S O(1,1)}
$$

instead of the coset space

$$
\frac{S L(2, \mathbb{R})}{S O(2)}
$$

of the IIB theory. The compactifications of these theories on spacelike tori $T^{n}$ and Lorentzian tori $T^{n, 1}$ (with $n$ spacelike circles and one timelike one) were considered in [2], generalising the results of $[12,13]$. In particular, whereas the type IIB theory is obtained from M-theory by reduction on $T^{2}$ in the limit in which the torus shrinks to zero size, the $I I B^{*}$ theory arises from M-theory compactified on $T^{1,1}$ in the limit in which it shrinks to zero size.

Given the existence of these 'new' type $I I^{*}$ theories, it is natural to ask whether any further new theories can be obtained in a similar way. This is indeed the case, and the purpose of this paper is to explore these new theories and the web of dualities linking them to each other and to M-theory. We start by pointing out two apparent problems arising from the findings of [2], the resolution of which leads to the first of the new theories. 
First, M-theory compactified on a timelike circle gives a string theory in 10 Euclidean dimensions which we will refer to as the $I I A_{E}$ theory (or $I I A_{10+0}$ theory). If this string theory is compactified on a circle, and the limit of zero radius is taken, the result should be the T-dual of the $I I A_{E}$ theory, which should again be a 10-dimensional string theory. The naive expectation is that it should again be in 10 Euclidean dimensions, and should be some Euclidean version of the type IIB theory. However, this cannot be the case, for a number of reasons. First, the IIB theory has chiral fermions and a 4-form gauge field with self-dual field strength, and there is no Euclidean version that does not double some of these degrees of freedom. Second, the supergravity limit of the T-dual of the $I I A_{E}$ theory should, when reduced to nine dimensions, give the same 9-dimensional theory as dimensionally reducing the $I I A_{E}$ theory; this 9-dimensional Euclidean theory is the one referred to as the $I I A_{9}$ theory in [2]. The only IIB-type supergravity theory in 10 dimensions compactifying to this theory is easily seen to be (up to field redefinitions) the $I I B^{*}$ theory compactified on a timelike circle, and the only Euclidean supergravity theory with this reduction (up to field redefinitions) is the $I I A_{E}$ theory itself.

Recall that the IIB string theory can be obtained by compactifying M-theory on a spacelike torus $T^{2}$ and taking the limit in which the torus shrinks to zero size [16]. M-theory on a torus with radii $R_{1}, R_{2}$ can be represented in the following diagram: 


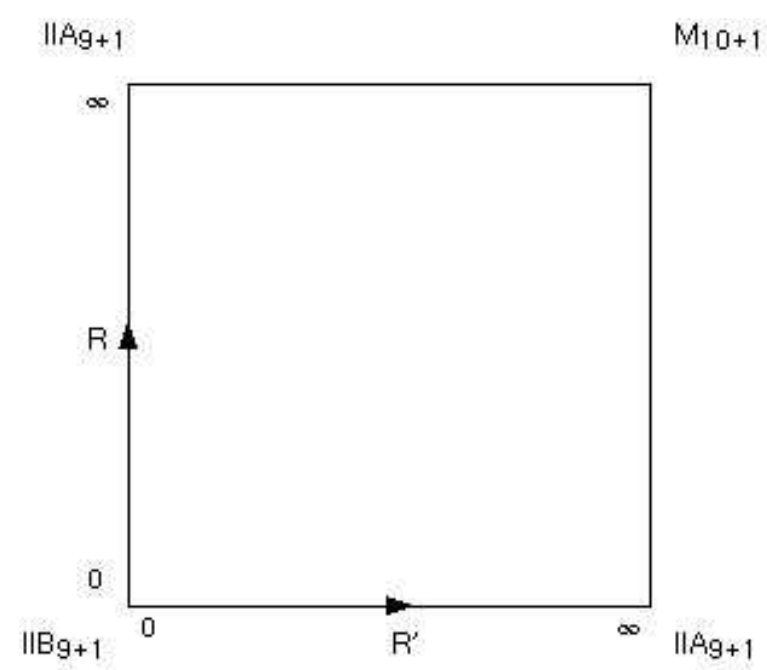

Figure 2 The moduli space for M-theory compactified on a rectangular torus $T^{2}$ with radii $R, R^{\prime}$. Points should be identified under the symmetry that interchanges $R$ with $R^{\prime}$.

If one of the radii shrinks, we get the weakly coupled IIA string and if both shrink, we obtain the IIB string with string coupling given by the ratio $R_{1} / R_{2}$.

Consider instead M-theory compactified on the Lorentzian torus $T^{1,1}$. First compactifying on a space-like circle gives type IIA string theory, and then compactifying on a timelike circle and taking the limit in which the circle shrinks to zero size gives the $I I B^{*}$ string theory, by timelike T-duality. If now the order of reductions is reversed, the reduction on the timelike circle gives the $I I A_{E}$ theory and a further reduction on a spacelike circle should again give the $I I B^{*}$ theory. This would lead one to expect a situation represented by the following diagram: 


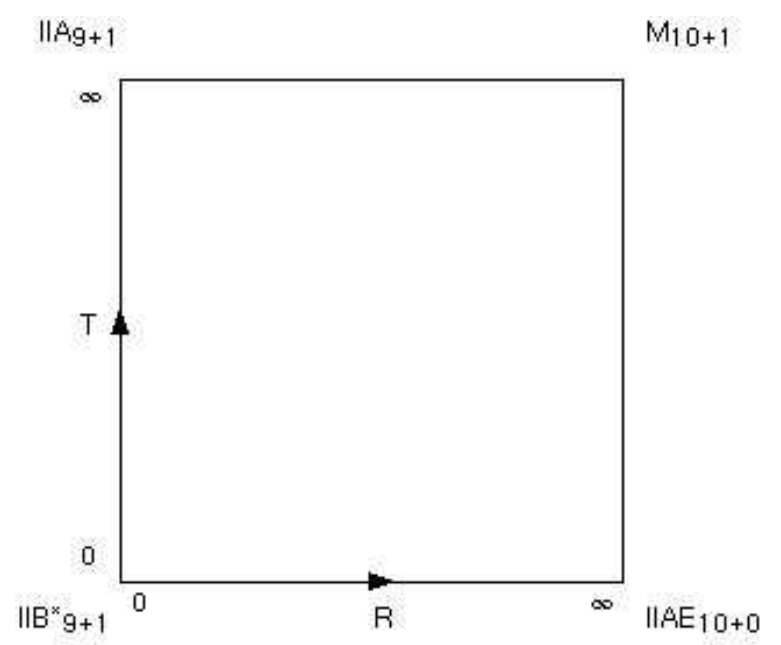

Figure 3 The moduli space for M-theory compactified on a Lorentzian torus $T^{1,1}$ with spacelike radius $R$ and timelike radius $T$.

Reducing on a $T^{1,1}$ with spacelike radius $R$ and timelike radius $T$ gives a moduli space of theories labelled by $R, T$ depicted in the diagram. If the order in which limits are taken does not matter, then the T-dual of the $I I A_{E}$ theory must be the $I I B^{*}$ theory. There are two obvious problems with this: (i) The T-duality would change the space-time signature, as it relates the $I I A_{E}$ theory in 10 Euclidean dimensions to the $I I B^{*}$ theory in $9+1$ dimensions with Lorentzian signature. The $I I A_{E}$ theory on a spacelike circle of radius $R$ is required to be T-dual to the IIB* theory on a timelike circle of radius $1 / R$. (ii) The $I I B^{*}$ theory already has a $\mathrm{T}$ dual, as the $I I B^{*}$ theory on a timelike circle of radius $1 / R$ is equivalent to the IIA theory on a timelike circle of radius $R$, and the $I I B^{*}$ theory on a spacelike circle is T-dual to the $I I A^{*}$ theory on a spacelike circle, so how can the $I I B^{*}$ theory be T-dual to the $I I A_{E}$ theory as well? On the other hand, if the order of the limits does matter, then the structure of the moduli space near the point $R=T=0$ could be rather complicated, as the different theories could arise, depending on the direction in which this point was approached. Also, if the II $B^{*}$ theory is not the T-dual of the $I I A_{E}$ theory, then it is hard to find another candidate. These issues will be resolved in section 4 and 5 , where it will be argued that under certain 
circumstances, T-duality can change a spacelike circle into a timelike one, and that the $I I B^{*}$ theory on a timelike circle is indeed T-dual to both the $I I A_{E}$ and $I I A^{*}$ theories. The point is that the string coupling constant of the $I I B^{*}$ theory is proportional to $R / T$, so that taking first $R$ small to obtain the weakly coupled IIA theory and then taking $T$ small to give the timelike T-dual of IIA gives the $I I B^{*}$ at weak coupling, while first taking $T$ small to give the weakly coupled $I I A_{E}$ theory and then taking $R$ small to give its T-dual results in the strong coupling limit of the $I I B^{*}$ string theory. The strong coupling limit of the $I I B^{*}$ string theory is, as will be seen, a different perturbative string theory, the $I I B^{\prime}$ theory, although the $I I B^{*}$ string theory and the $I I B^{\prime}$ string theory are different perturbative limits of the same underlying non-perturbative theory. The timelike T-dual of the II $B^{*}$ string theory is the $I I A$ string theory, as expected, but the timelike T-dual of the $I I B^{\prime}$ string theory is the $I I A_{E}$ string theory. However, having resolved this issue, we find the T-dual of the $I I B^{\prime}$ theory on a space-like circle leads to something new again, as will be discussed in section 6 .

A second open question is what is the strong coupling limit of the $I I A^{*}$ string theory. As in the IIA case, it is expected to be some 11-dimensional theory. However, it cannot have the usual 11-dimensional supergravity as its field theory limit, as the reduction of this gives the $I I A$ theory, not the $I I A^{*}$ theory. The natural guess is that it should have a $10+1$ dimensional field theory limit, but we will see that there is no such theory which gives the right reduction. This will be addressed in section 7 , and the strong coupling limit will be identified as a theory in $9+2$ dimensions, and a field theory in $9+2$ dimensions which dimensionally reduces to the $I I A^{*}$ theory will be found. 


\section{Timelike T-duality}

Consider first the bosonic or heterotic string. For a flat string theory background which includes a periodic coordinate $X$, the most general string configuration in that direction $X(\sigma, \tau)$ is

$$
X(\sigma, \tau)=x+p \tau+\tilde{p} \sigma+\sum_{n}\left(a_{n} e^{i n(\tau+\sigma)}+\tilde{a}_{n} e^{i n(\tau-\sigma)}\right)
$$

where $\tau, \sigma$ are the closed-string world-sheet coordinates, with periodic coordinate $\sigma \sim \sigma+2 \pi$, and $\tau \in \mathbb{R}$. If $X$ is periodically identified $X \sim X+2 \pi R$ for some radius $R$, then the phase $e^{i p \cdot x}$ will be single-valued if the momentum $p$ is quantized

$$
p=\frac{n}{R}
$$

with $n$ an integer, while the dual momentum $\tilde{p}$ must satisfy

$$
\tilde{p}=m R
$$

so that $X$ is well-defined. Then $m$ is the number of times the periodic coordinate $\sigma$ of the string world-sheet winds around the $X$ dimension. The physical state conditions are invariant under the T-duality transformation that interchanges $m$ and $n$ and takes $R \rightarrow 1 / R$ and so interchanges $p$ with $\tilde{p}$. Writing

$$
X(\tau, \sigma)=X(\tau+\sigma)+\tilde{X}(\tau-\sigma)
$$

the T-duality takes $X \rightarrow X$, and $\tilde{X} \rightarrow-\tilde{X}$.

In the above, the compact coordinate $X$ can be either spacelike or timelike, and if it is timelike, $X=X^{0}$, then $p=p^{0}$ is the energy, which is quantized, $p^{0}=n / R$, and $m$ is the number of times the $\sigma$ coordinate on the world-sheet winds around the timelike direction in the target space. For a configuration in which the compact world-sheet coordinate $\sigma$ maps to the target space time $X^{0}$, 
the non-compact world-sheet coordinate $\tau$ would map to a spatial direction $X^{1}$, say, in the target space, if the world-sheet is to be non-degenerate. Note that for such configurations, $\tau$ becomes a space coordinate and $\sigma$ a periodic time coordinate with respect to the induced world-sheet metric. Then $p^{\mu}=\partial_{\tau} X^{\mu}$ is a tachyonic (spacelike) momentum, and it is straightforward to see that such states must occur. Consider, for example, a conventional string state with timelike momentum $\left(p^{0}=\right.$ $\left.n / R, p^{i}\right)$ and no winding number. Then a timelike T-duality would take this to a state with timelike winding number $n$ and momentum $\left(0, p^{i}\right)$, which is spacelike and tachyonic. Nonetheless, this is the original 'good' state described in terms of dual variables, so that the unusual features are a consequence of the variables used and do not reflect any pathology of the original state. For the heterotic string compactified on time to $\mathbb{R}^{9} \times S^{1}$, both the limits $R \rightarrow \infty$ and $R \rightarrow 0$ give the heterotic string in $9+1$ dimensional Minkowski space which is free from both ghosts and tachyons (at least perturbatively) and is stable. For the tachyonic state described above, the winding number becomes the energy corresponding to the dual time coordinate, and the resulting dual momentum is timelike, as it should be. The perturbative spectrum for finite $R$ has been analysed in [1], where it was shown to be free from ghosts, with a positive definite Hilbert space.

Consider the low-energy field theory, which includes

$$
S=\int d^{10} x \sqrt{-g} e^{-2 \Phi}\left(R+4(\partial \Phi)^{2}-H^{2}\right)+\ldots
$$

where $\Phi$ is the dilaton and $H=d B_{2}$ is the field strength for the 2-form gauge field $B_{2}$. Reducing this on time to 9 Euclidean dimensions gives an action

$$
S=\int d^{9} x \sqrt{g}\left[e^{-2 \Phi}\left(R+4(\partial \Phi)^{2}-H^{2}-d \chi^{2}+F_{g}^{2}+F_{B}^{2}\right)+\ldots\right]
$$

where $F_{g}, F_{B}$ are the field strengths for the one-form gauge fields $A_{i}^{g} \sim g_{i 0}, A_{i}^{B} \sim$ $B_{i 0}$ and $\chi$ is the scalar coming from the metric. The T-duality leads to a symmetry of the 9-dimensional action under which $A^{g}, A^{B}$ are interchanged. The kinetic 
terms for the two vector fields $A^{g}, A^{B}$ have the 'wrong' sign, usually associated with ghosts. However, if all the Kaluza-Klein modes are kept, the theory is the full 10-dimensional theory and the 10-dimensional gauge symmetries can be used to remove all negative norm states, including those corresponding to the fields $g_{i 0}, B_{i 0}$, at least in the topologicaly trivial sector. The 10-dimensional physical states carry non-zero $p^{0}$ and all of these are thrown away in the truncation of dependence on the $X^{0}$ coordinate, so that the dimensionally reduced action (3.6) is particularly misleading for studying the physical states. Nonetheless, such actions can be useful for studying some aspects of the theory, and relations between theories, but should be regarded, for most purposes, as being accompanied by an action for an infinite set of modes with non-zero energy, and most of the physics is in these modes.

Consider now T-duality in the type II theories, which in the covariant NSR formalism are formulated in terms of bosonic coordinates $X^{\mu}$ and their superpartners $\Psi^{\mu}$, which are world-sheet spinors. The transformation $\partial_{a} X^{\mu} \rightarrow \epsilon_{a}^{b} \partial_{b} X^{\mu}$ or $X^{\mu}+\tilde{X}^{\mu} \rightarrow X^{\mu}-\tilde{X}^{\mu}$ for T-duality in the $\mu$ direction is accompanied by the transformation $\Psi^{\mu} \rightarrow \gamma^{3} \Psi^{\mu}$. Decomposing $\Psi^{\mu}$ into a right-handed Majorana-Weyl world-sheet fermion $\psi^{\mu}$ and a left-handed one $\tilde{\psi}^{\mu}$, this transformation acts as

$$
\psi^{\mu} \rightarrow \psi^{\mu}, \quad \tilde{\psi}^{\mu} \rightarrow-\tilde{\psi}^{\mu}
$$

In addition, there are right-handed and left-handed spin operators $S, \widetilde{S}$ which are Majorana space-time spinors satisfying the chirality constraints $\Gamma^{11} S=S, \Gamma^{11} \tilde{S}=$ $\tilde{S}$ for the type IIB string and $\Gamma^{11} S=S, \Gamma^{11} \tilde{S}=-\tilde{S}$ for the type IIA string [14]. As a reflection in a spatial direction, $X^{9}$ say, acts in spin space through the operator $i \Gamma^{11} \Gamma^{9}$, T-duality in the $X^{9}$ direction acts on the spin operators as [15]

$$
S \rightarrow S, \quad \tilde{S} \rightarrow \tilde{S}^{\prime}=i \Gamma^{11} \Gamma^{9} \tilde{S}
$$

This changes the chirality of $\tilde{S}$. By considering the action on RR vertex operators [15], this gives the relation between the RR field strengths $G_{n}^{\prime}$ of the type IIA 
(IIB) theory with $n$ even (odd), and the field strengths $G_{n \pm 1}$ of the T-dual type IIB (IIA) theory:

$$
G_{\mu_{1} \ldots \mu_{n}}^{\prime}=G_{9 \mu_{1} \ldots \mu_{n}}, \quad G_{9 \mu_{1} \ldots \mu_{n}}^{\prime}=-G_{\mu_{1} \ldots \mu_{n}}
$$

for any $\mu_{i} \neq 9$.

For a timelike T-duality in the $X^{0}$ direction, the T-duality should take $\tilde{S} \rightarrow P \tilde{S}$ for some operator $P$ which should be given by $\Gamma^{11} \Gamma^{0}$, up to a phase. There are possible phases (up to a sign), which lead to equivalent results. The operator $P=i \Gamma^{11} \Gamma^{0}$ preserves the Majorana condition on $\tilde{S}$ but is not unitary, while $\Gamma^{11} \Gamma^{0}$ is unitary but does not preserve the Majorana condition. Choosing the unitary operator, then (in a Majorana representation in which Majorana spinors are real and the gamma matrices imaginary) $\tilde{S}^{\prime}$ is imaginary. As a result, a timelike Tduality maps the type II theory with real (Majorana) spin operators $S, \tilde{S}$ to a new theory, the type $I I^{*}$ theory, in which there is a real right-handed spin operator $S$ and an imaginary left-handed one $\hat{S}$, with the T-duality taking $\tilde{S} \rightarrow \hat{S}=\Gamma^{11} \Gamma^{0} S$. If we choose instead $P=i \Gamma^{11} \Gamma^{0}$, the T-duality preserves the reality of $\tilde{S}$ and changes its chirality, but the fields in the R-R and NS-R sectors (i.e. the sectors constructed using $\tilde{S}$ ) become imaginary, so that the timelike T-dual of the IIA (IIB) theory is given by acting on the IIB (IIA) theory with $i^{F_{L}}$ to give the $I I B^{*}$ $\left(I I A^{*}\right)$ string theory. This has the effect of multiplying all fields in the NS-R and $\mathrm{R}$-R sectors by $i$, so that on rewrting in terms of real fields, the signs of all terms in the action that are bilinear in such fields, including the kinetic terms, are reversed, and exactly the same sign reversals arise from calculating the effective action in the formalism based on the imaginary spin operator $\hat{S}$, but with real fields. In either approach, the RR field strengths $G_{n}$ of the type IIA (IIB) theory with $n$ even (odd) are related to the field strengths $\hat{G}_{n \pm 1}$ of the T-dual type $I I B^{*}\left(I I A^{*}\right)$ theory by

$$
G_{\mu_{1} \ldots \mu_{n}}=\hat{G}_{0 \mu_{1} \ldots \mu_{n}}, \quad G_{0 \mu_{1} \ldots \mu_{n}}=-\hat{G}_{\mu_{1} \ldots \mu_{n}}
$$

for any $\mu_{i} \neq 0$. 
An important check on these signs and phases is given by the supergravity theories. The T-duality between the IIA and IIB theories is reflected in the supergravity theories by the fact that the dimensional reduction of IIA supergravity to $8+1$ dimensions is equivalent to the reduction of IIB supergravity on a spacelike circle, and the field redefiniton relating the two theories gives the T-duality rules, and in particular gives the RR field transformations (3.9) [18], together with the transformations found by Buscher [20]. Similarly, the IIA and IIB* supergravities have the same timelike reduction to $9+0$ dimensions, as do the IIB and $I I A^{*}$ supergravities, and the field redefinitions linking them give the T-duality transformations.

If there are fundamental strings whose world-sheets wrap around a compact time dimension, then there must be branes whose world-volumes also wrap around compact time. For example, a fundamental string of the IIB string theory winding in time is S-dual to a D-string winding time, and spatial T-duality leads to $\mathrm{D} p$ branes of the IIB or IIA theories which also wrap around the time dimension. The S-dual of a wrapped D5-brane gives a wrapped IIB NS 5-brane, and T-dualities relate these to wrapped NS-5-branes and KK monopoles of the IIA and IIB theories. It was argued in [19] that time-wrapping branes allows one to avoid singularities and certain other undesirable features in the brane supergravity solutions.

A timelike T-duality takes a time-wrapped $\mathrm{D} p$-brane (with $p+1$ dimensional Lorentzian world-volume) of the type IIA (IIB) theory to an Ep-brane of the type $I I B^{*}\left(I I A^{*}\right)$ string theory with a $p$-dimensional Euclidean world-volume [2]. For example, a time-wrapped D-string is T-dual to an E1-brane of the $I I A^{*}$ theory whose world-line is spacelike and so can be thought of as the world-line of a tachyon, but this tachyon is closely related to the tachyon discussed above, arising from T-dualising a time-wrapped fundamental string. The $I I B^{*}$ theory has fundamental strings with Lorentzian world-sheets and E2-branes or 'Euclidean strings' with Euclidean 2-dimensional world-sheets, but the two are interchanged by $S L(2, \mathbb{Z})$ Sduality transformations. This duality with normal branes suggests that the tachyonic branes might not be as problematic as they at first appear. As will be seen, 
there can be supersymmetric string theories in which the 'fundamental strings' are Euclidean, with Euclidean world-sheets embedded in a Lorentzian target space. Similar considerations lead to branes of various world-volume signatures occurring in the M-theories and strings of various signatures [3] and dualities link these, so all should be on the same footing.

\section{T-Dual of the $I I A_{E}$ Theory}

The $I I A_{E}$ string theory is the timelike reduction of M-theory, and is in 10 Euclidean dimensions. The fundamental strings also have Euclidean world-sheets, as they arise from double timelike dimensional reduction of M2-branes. In this section, we will seek the T-dual of the II $A_{E}$ theory; it will be useful to denote it as the $I I B^{\prime}$ theory. We will start by looking at the supergravity effective actions, and identify the perturbative branes as in [17]. In particular, the dimensional reduction of the $I I B^{\prime}$ theory to 9 Euclidean dimensions should be the same as that of the II $A_{E}$ theory.

The bosonic action of the IIA supergravity is

$$
\begin{aligned}
& S_{I I A}=\int d^{10} x \sqrt{-g}\left[e^{-2 \Phi}\left(R+4(\partial \Phi)^{2}-H^{2}\right)\right. \\
&\left.-G_{2}^{2}-G_{4}^{2}\right]+\frac{4}{3} \int G_{4} \wedge G_{4} \wedge B_{2}+\ldots
\end{aligned}
$$

while that of IIB supergravity is

$$
\begin{gathered}
S_{I I B}=\int d^{10} x \sqrt{-g}\left[e^{-2 \Phi}\left(R+4(\partial \Phi)^{2}-H^{2}\right)\right. \\
\left.-G_{1}^{2}-G_{3}^{2}-G_{5}^{2}\right]+\ldots
\end{gathered}
$$

Here $\Phi$ is the dilaton, $H=d B_{2}$ is the field strength of the NS-NS 2-form gauge field $B_{2}$ and $G_{n+1}=d C_{n}+\ldots$ is the field strength for the RR $n$-form gauge field $C_{n}$. The field equations derived from the IIB action (4.2) are supplemented with the self-duality constraint $G_{5}=* G_{5}$. 
The corresponding actions for the type $I I^{*}$ theories have RR kinetic terms with the opposite sign [2]:

$$
\begin{aligned}
S_{I I A^{*}}=\int d^{10} x & \sqrt{-g}\left[e^{-2 \Phi}\left(R+4(\partial \Phi)^{2}-H^{2}\right)\right. \\
& \left.+G_{2}^{2}+G_{4}^{2}\right]-\frac{4}{3} \int G_{4} \wedge G_{4} \wedge B_{2}+\ldots
\end{aligned}
$$

and

$$
\begin{gathered}
S_{I I B^{*}}=\int d^{10} x \sqrt{-g}\left[e^{-2 \Phi}\left(R+4(\partial \Phi)^{2}-H^{2}\right)\right. \\
\left.+G_{1}^{2}+G_{3}^{2}+G_{5}^{2}\right]+\ldots
\end{gathered}
$$

The type $I I B^{*}$ theory has a scalar coset space

$$
\frac{S L(2, \mathbb{R})}{S O(1,1)}
$$

and enjoys an $S L(2, \mathbb{Z})$ U-duality, and again $G_{5}=* G_{5}$.

Reducing M-theory on a timelike $S^{1}$ gives the $I I A_{E}$ string theory, and this has a supergravity limit given by the reduction of 11-dimensional supergravity on a timelike circle. This is a theory in 10 Euclidean dimensions with bosonic action

$$
\begin{aligned}
S_{I I A_{E}}=\int d^{10} x & \sqrt{g}\left[e^{-2 \Phi}\left(R+4(\partial \Phi)^{2}+H^{2}\right)\right. \\
& \left.+G_{2}^{2}-G_{4}^{2}\right]+\frac{4}{3} \int G_{4} \wedge G_{4} \wedge B_{2}+\ldots
\end{aligned}
$$

Reducing the type IIB or $I I A^{*}$ theory in the time direction gives the $I I B_{9}$ theory in 9 Euclidean dimensions with bosonic kinetic terms

$$
\begin{gathered}
S_{I I B_{9}}=\int d^{9} x \sqrt{g}\left[e^{-2 \Phi}\left(R+4(\partial \Phi)^{2}-H^{2}-d \chi^{2}+F_{g}^{2}+F_{B}^{2}\right)\right. \\
\left.-G_{1}^{2}+G_{2}^{2}-G_{3}^{2}+G_{4}^{2}\right]+\ldots
\end{gathered}
$$

and scalars taking values in

$$
\frac{S L(2, \mathbb{R})}{S O(2)} \times \mathbb{R}^{+}
$$


while the timelike reduction of the IIA or II $B^{*}$ theory gives the $I I A_{9}$ theory

$$
\begin{gathered}
S_{I I A_{9}}=\int d^{9} x \sqrt{g}\left[e^{-2 \Phi}\left(R+4(\partial \Phi)^{2}-H^{2}-d \chi^{2}+F_{g}^{2}-F_{B}^{2}\right)\right. \\
\left.+G_{1}^{2}-G_{2}^{2}+G_{3}^{2}-G_{4}^{2}\right]+\ldots
\end{gathered}
$$

with scalars taking values in

$$
\frac{S L(2, \mathbb{R})}{S O(1,1)} \times \mathbb{R}^{+}
$$

The fact that the timelike dimensional reduction of the IIB and $I I A^{*}$ (or IIA and $\left.I I B^{*}\right)$ theories gives the same 9-dimensional theory shows that timelike T-duality should relate the two theories [2].

On the other hand, reducing the $I I A_{E}$ supergravity to 9 dimensions gives the bosonic action

$$
\begin{gathered}
S_{I I A_{9}^{\prime}}=\int d^{9} x \sqrt{g}\left[e^{-2 \Phi}\left(R+4(\partial \Phi)^{2}+H^{2}-d \chi^{2}+F_{g}^{2}+F_{B}^{2}\right)\right. \\
\left.+G_{1}^{2}+G_{2}^{2}-G_{3}^{2}-G_{4}^{2}\right]+\ldots
\end{gathered}
$$

with scalars again taking values in (4.10). This type $I I A_{9}^{\prime}$ theory is related to the type $I I A_{9}$ theory by a field redefinition, which is an $S L(2, \mathbb{Z})$ transformation interchanging $B_{2}$ with $C_{2}$ and $C_{1}$ with $A^{g}$. The dimensional reduction of 11dimensional supergravity on space and then time gives the $I I A_{9}$ supergravity, while reducing on time and then space gives the II $A_{9}^{\prime}$ supergravity. These two 9dimensional supergravities are related by a field redefinition and so are equivalent, so that reducing on time and then space gives the same result as reducing on space and then time, as in [13]. The $I I A_{9}^{\prime}$ theory can also be obtained by the timelike dimensional reduction of the following $9+1$ dimensional bosonic action, which we will refer to as the $I I B^{\prime}$ action:

$$
\begin{gathered}
S_{I I B^{\prime}}=\int d^{10} x \sqrt{-g}\left[e^{-2 \Phi}\left(R+4(\partial \Phi)^{2}+H^{2}\right)\right. \\
\left.+G_{1}^{2}-G_{3}^{2}+G_{5}^{2}\right]+\ldots
\end{gathered}
$$

This differs from the $I I B^{*}$ action (4.4) in the signs of the kinetic terms of the 2-form gauge fields: the NS-NS 2-form $B_{2}$ has a kinetic term of the right sign in 
the $I I B^{*}$ theory and the wrong sign in the $I I B^{\prime}$ action, while the $\mathrm{RR} 2$-form $C_{2}$ has a kinetic term of the wrong sign in the $I I B^{*}$ theory and the right sign in the $I I B^{\prime}$ action.

The strong-coupling limit of the $I I B^{*}$ theory is given by acting with an $S L(2, \mathbb{Z})$ transformation that takes $\Phi \rightarrow-\Phi$ (if $C_{0}=0$ ) and which interchanges $B_{2}$ and $C_{2}$ so that, after a Weyl-rescaling of the metric, the $I I B^{*}$ action (4.4) is replaced by the $I I B^{\prime}$ action (4.12), and the strong coupling limit of the $I I B^{*}$ string theory is the $I I B^{\prime}$ string theory with field-theory limit (4.12).

This leads to the following scenario. The T-dual of the $I I A_{E}$ theory is a $I I B^{\prime}$ string theory whose zero-slope limit is the $I I B^{\prime}$ supergravity (4.12), which is related to the $I I B^{*}$ supergravity by the field redefinition interchanging $B_{2}$ and $C_{2}$, so that the $I I B^{\prime}$ and $I I B^{*}$ supergravities are equivalent field theories, with two 2-form gauge fields which form an $S L(2)$ doublet and have kinetic terms of opposite signs. However, the $I I B^{\prime}$ and $I I B^{*}$ theories are different perturbative string theories. The 2 -form with the right-sign kinetic term couples to strings with $1+1$ dimensional world-sheets in the usual way (the fundamental strings of the $I I B^{*}$ theory) while the one with the wrong-sign kinetic term couples to 'Euclidean strings' whose world-sheets are spacelike surfaces with 2 Euclidean dimensions embedded in Lorentzian spacetime (the E2-branes of the $I I B^{*}$ theory). Thus the $I I B^{*}$ and the $I I B^{\prime}$ theories each have both Lorentzian and Euclidean strings, and which of the various branes are the perturbative states can be seen using the arguments of [17]. The weakly coupled $I I B^{*}$ theory is a perturbative theory of the Lorentzian strings, with the Euclidean strings arising as non-perturbative E-branes, while in the $I I B^{\prime}$ theory, the roles are reversed, and the theory is a perturbative theory of the Euclidean strings with the Lorentzian strings arising in the non-perturbative sector as D-strings on which the Euclidean strings can end.

Thus, at the non-perturbative level, there is a theory in $9+1$ dimensions which has both strings with Lorentzian world-sheets and strings with Euclidean worldsheets, and whose zero-slope limit is the type $I I B^{*}$ supergravity with bosonic 
action (4.4) (or, equivalently, the type $I I B^{\prime}$ supergravity (4.12)). The perturbative string theory based on the Lorentzian strings is the type $I I B^{*}$ string theory whose timelike T-dual is the type $I I A^{*}$ string theory, while the perturbative string theory based on the Euclidean strings is the type $I I B^{\prime}$ string theory whose timelike T-dual is the type $I I A_{E}$ string theory, which is again a theory of Euclidean world-sheets. The $I I B^{*}$ and $I I B^{\prime}$ string theories are S-dual, so one is the strong-coupling limit of the other. We see that the T-dual of the $I I A_{E}$ string theory is not a version of the IIB theory with Euclidean target space, but a version with Euclidean world-sheet instead. We also learn that the diagram in figure 3 is essentially correct, so long as the $I I B^{*}$ theory occurring at $R=T=0$ is taken as the full non-perturbative theory; the coupling constant is given by the ratio $R / T$, as in [16], and so taking $R$ to zero and then $T$ to zero gives the $I I B^{*}$ string theory while reversing the order gives its strong coupling limit, the $I I B^{\prime}$ string.

The key feature here is that the 2-form with the wrong sign kinetic term couples to strings with Euclidean world-sheets, and a Euclidean string on a spacelike circle of radius $R$ is T-dual to a Euclidean string theory on a timelike circle of radius $1 / R$, so that whereas T-duality of Lorentzian strings does not change the spacetime signature, T-duality of Euclidean ones does. In the next section, we will show why this is the case; in later sections, this will lead to further circumstances in which dualities can change space-time signature.

\section{Euclidean Strings and T-Duality}

Consider a string theory with a flat target space with a circular dimension with coordinate $X \sim X+1$, so that the world-sheet action includes

$$
S=\lambda \frac{1}{2} \int d^{2} \sigma \partial_{a} X \partial^{a} X+\ldots
$$

where $\lambda=R^{2}$ for a space-like circle of radius $R$ and $\lambda=-R^{2}$ for a timelike circle of radius $R$, and $\sigma^{a}$ are the world-sheet coordinates. T-duality can be studied by 
gauging the shift symmetry $X \rightarrow X+c$ by coupling to a world-sheet gauge field $A_{a}$ and adding a term with a Lagrange multiplier field $Y$ imposing the constraint that the gauge field is flat [20-24]. This gives the action

$$
S=\int d^{2} \sigma\left(\frac{1}{2} \lambda D_{a} X D^{a} X+\epsilon^{a b} A_{a} \partial_{b} Y+\ldots\right)
$$

where

$$
D_{a} X=\partial_{a} X-A_{a}
$$

Then the Lagrange multiplier imposes the constraint $F_{a b}=0\left(F_{a b}=\partial_{a} A_{b}-\partial_{b} A_{a}\right)$ and implies $A$ is pure gauge and can be gauged away, recovering the action (5.1). The multiplier field $Y$ takes values in a circle, $Y \sim Y+1$, so that the winding modes in $Y$ are responsible for the elimination of flat connections with non-trivial holonomy. On the other hand, the terms involving $X$ can be gauged away, after which $A_{a}$ is an auxiliary field which can be eliminated to give

$$
S=\lambda^{\prime} \frac{1}{2} \int d^{2} \sigma \partial_{a} Y \partial^{a} Y+\ldots
$$

where

$$
\lambda^{\prime}=-\frac{\varepsilon}{\lambda}
$$

and $\varepsilon$ is the coefficient in the identity

$$
\epsilon^{a b} \epsilon^{c d}=\varepsilon\left(h^{a c} h^{b d}-h^{a d} h^{b c}\right)
$$

where $h_{a b}$ is the world-sheet metric. Then $\varepsilon=-1$ for a Lorentzian world-sheet and $\varepsilon=+1$ for a Euclidean world-sheet. Thus for a Lorentzian world-sheet, $\lambda^{\prime}=1 / \lambda$ and a spacelike (timelike) circle of radius $R$ in which $X$ takes values has been exchanged for the dual spacelike (timelike) circle of radius $1 / R$ in which $Y$ takes values, so the signature is unchanged. On the other hand, for a Euclidean world-sheet, $\lambda^{\prime}=-1 / \lambda$ and a spacelike (timelike) circle of radius $R$ in which $X$ 
takes values has been exchanged for the dual timelike (spacelike) circle of radius $1 / R$ in which $Y$ takes values, so the signature of the $X$ coordinate is reversed. The T-duality corresponds to the map $X \rightarrow Y$ where $\partial_{a} X=\epsilon_{a}^{b} \partial_{b} Y$, so that $(\partial X)^{2}=-\varepsilon(\partial Y)^{2}$.

More generally, consider a non-linear sigma-model

$$
S=\frac{1}{2} \int d^{2} \sigma \sqrt{|h|}\left(g_{m n} \partial_{a} X^{m} \partial^{a} X^{n}+B_{m n} \epsilon^{a b} \partial_{a} X^{m} \partial_{b} X^{n}+\Phi R\right)
$$

representing a string moving in a target space with coordinates $X^{m}$, metric $g_{m n}$ and background 2-form gauge field $B_{m n}$ and dilaton $\Phi$. Suppose the target space has an isometry generated by a Killing vector $k^{m}$ and that the Lie derivatives of $H_{m n p}$ and the dilaton vanish. Then there is a symmetry $\delta X=c k^{m}$ for constant parameter $c$ and this can be gauged if the global obstruction of [25] is absent. In that case, a Lagrange multiplier term can again be added to the gauged action to obtain [20-24]

$$
S=S_{\text {gauged }}\left[X^{m}, A_{a}\right]+\int d^{2} \sigma \epsilon^{a b} A_{a} \partial_{b} Y
$$

where $S_{\text {gauged }}$ is the gauged action given in [25]. If the isometry orbits are compact, the Lagrange multiplier can again be eliminated to regain the action (5.7), or one can integrate over the gauge fields to obtain the T-dual sigma-model with background fields $\tilde{g}_{m n}, \tilde{B}_{m n}, \tilde{\Phi}$. Choosing adapted coordinates $X^{m}=\left(X^{0}, X^{\alpha}\right)$ where $k=\partial / \partial X^{0}$ so that the isometry generates shifts in $X^{0}$, the dual background

$\star$ We use the notation of [20-24], but do not specify here whether $X^{0}$ is a spacelike or timelike coordinate. 
fields are

$$
\begin{aligned}
\tilde{g}_{00} & =-\varepsilon \frac{1}{g_{00}} \\
\tilde{g}_{0 \alpha} & =-\varepsilon \frac{B_{0 \alpha}}{g_{00}} \\
\tilde{B}_{0 \alpha} & =\frac{g_{0 \alpha}}{g_{00}} \\
\tilde{g}_{\alpha \beta} & =g_{\alpha \beta}-\frac{g_{0 \alpha} g_{0 \beta}-(-\varepsilon) B_{0 \alpha} B_{0 \beta}}{g_{00}} \\
\tilde{B}_{\alpha \beta} & =B_{\alpha \beta}-\frac{g_{0 \alpha} B_{0 \beta}-B_{0 \alpha} g_{0 \beta}}{g_{00}} \\
\tilde{\Phi}=\Phi-\frac{1}{2} \log \left|g_{00}\right| &
\end{aligned}
$$

These are the same as the transformations of [20], except that the factors of $-\varepsilon$, with $\varepsilon=-1$ for Minkowski world-sheets and $\varepsilon=1$ for Euclidean world-sheets, changes some of the signs for Euclidean strings. In particular, as $\tilde{g}_{00}=-\varepsilon / g_{00}$, the target space signature changes if $\varepsilon=1$.

The metric beta-function for the sigma-model (5.7) is

$$
\beta_{m n}^{g}=R_{m n}-(-\varepsilon) H_{m p q} H_{n}^{p q}+\ldots
$$

as the identity (5.6) is used in the Feynman graphs leading to the $H^{2}$ term. As a result, the effective action generating the conditions for sigma-model conformal invariance is

$$
S=\int d^{D} X \sqrt{|g|} e^{-2 \Phi}\left(R+4 \nabla \Phi^{2}-(-\varepsilon) H^{2}\right)+\ldots
$$

and the sign of the $B_{2}$ kinetic term is indeed reversed for Euclidean world-sheets $(\varepsilon=1)$, as expected from the last section.

Note that the Euclidean strings considered here should be distinguished from the Wick-rotated strings used in Euclidean path integrals. Consider for example the sigma-model (5.7) with Lorentzian world-sheet and Lorentzian target space. In 
the Euclidean path integral quantization, the target space is (usually) Wick-rotated or analytically continued to a Euclidean space and the path integral is taken to be over all Euclidean world-sheet metrics. The functional integral is weighted with $e^{-S_{E}}$ where $S_{E}$ is the Euclideanised action which in particular has an imaginary Wess-Zumino term:

$$
S_{E}=\frac{1}{2} \int d^{2} \sigma \sqrt{|h|}\left(g_{m n} \partial_{a} X^{m} \partial^{a} X^{n}+i B_{m n} \epsilon^{a b} \partial_{a} X^{m} \partial_{b} X^{n}+\Phi R\right)
$$

The factor of $i$ in front of the Wess-Zumino term is needed to make the functional integral well-defined for topologically non-trivial $B_{2}$ fields and gives an extra sign to cancel the one coming from the identity (5.6) on changing from Lorentzian to Euclidean world-sheets. The resulting target space effective action is then

$$
S=\int d^{D} X \sqrt{|g|} e^{-2 \Phi}\left(R+4 \nabla \Phi^{2}-H^{2}\right)+\ldots
$$

with the correct signs, and the T-duality transformations are those of Buscher, without any sign-changes. The action for the Euclidean strings that was considered above has a real Wess-Zumino term and so has some signs changed in the T-duality rules and the effective action. Formally, one can think of using the action (5.7) in a path-integral weighted with $e^{i S}$, and then consider various Wick rotations or analytic continuations as appropriate to define the functional integral; these include Wick rotations of timelike target space coordinates $X$ as well as the world-sheet time. 


\section{More Type II String Theories}

The type $I I B^{\prime}$ theory has Euclidean fundamental strings and so, by the arguments of the last section, T-duality changes the signature. Compactifying on a timelike circle and T-dualising gives the $I I A_{E}$ theory in 10 Euclidean dimensions, while using a spacelike circle will give a theory in $8+2$ dimensions. The supergravity action for the theory in $8+2$ dimensions is determined by requiring its timelike reduction to $8+1$ dimensions be the same as the spacelike reduction of the $I I B_{9+1}^{\prime}$ supergravity, and is again of the type IIA form but with certain sign changes. The theory in $8+2$ dimensions will be referred to as the type $I I A_{8+2}$ theory. Both the $I I A_{E}$ and $I I A_{8+2}$ theories have a $B_{2}$ field with the wrong sign kinetic term and so both have Euclidean fundamental strings.

In the same way, a spacelike T-duality of the $I I A_{8+2}$ theory will give a $I I B_{7+3}$ theory in $7+3$ dimensions which has a type IIB-style action but with some sign changes (given in table 1 of section 12). It has scalars in the coset space $S L(2, \mathbb{R}) / S O(2)$, and both the 2 -forms $C_{2}$ and $B_{2}$ have kinetic terms of the wrong sign, so that there are $(p, q)$ strings with Euclidean world-sheets. A spacelike Tduality then takes this to a $I I A_{6+4}$ theory in 6 spatial and 4 time dimensions, and a further spacelike T-duality takes this to a IIB-like theory in $5+5$ dimensions, which will be denoted the $I I B_{5+5}^{\prime}$ string theory, as it is similar to the $I I B_{9+1}^{\prime}$ theory. It has fundamental Euclidean strings and Lorentzian D-strings, and the strong coupling limit is a type $I I B_{5+5}^{*}$ string theory with perturbative Lorentzian strings and E2-branes (or Euclidean D-strings). This has a $I I A_{5+5}^{*}$ theory as a spacelike T-dual, and a $I I A_{5+5}$ theory as a timelike T-dual, and finally the $I I A_{5+5}$ has a $I I B_{5+5}$ theory as a spacelike T-dual, and this latter is the timelike T-dual of the $I I A_{5+5}^{*}$ theory. The T-dualities relating the theories in $5+5$ dimensions are shown in figure 4 , and is very similar to the $9+1$ dimensional case depicted in figure 1 . 


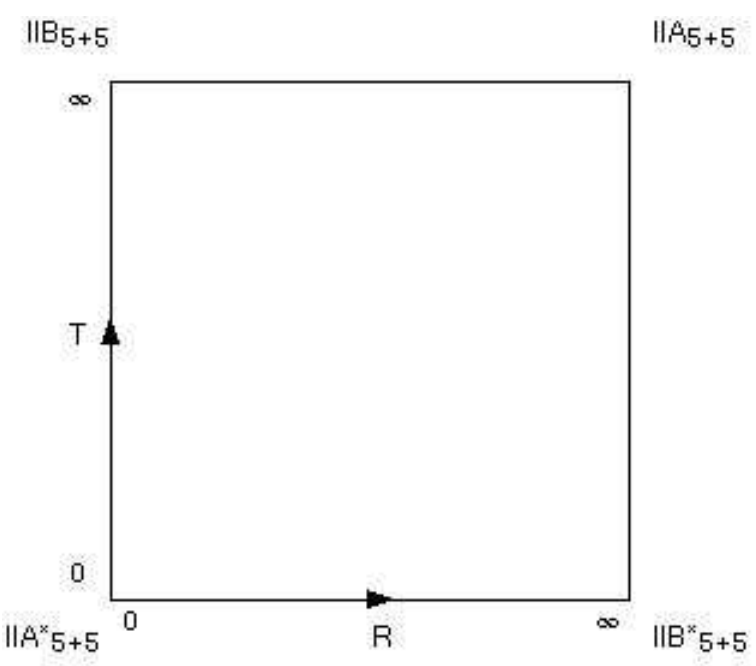

Figure 4 The moduli space for the type $I I A_{5+5}$ theory compactified on a Lorentzian torus with timelike radius $T$ and spacelike radius $R$.

Spacelike T-duality relates the IIA and IIB theories and the $I I A^{*}$ and $I I B^{*}$ theories, while timelike T-duality relates the $I I A^{*}$ and IIB theories and the IIA and $I I B^{*}$ theories. The type $I I B_{5+5}^{*}$ string and the type $I I B_{5+5}^{\prime}$ string are different perturbative limits of the same non-perturbative theory. After this, further T-dualities give a set of theories similar to those just listed but with space and time interchanged. Thus there are IIA,IIB,II $A^{*} I I B^{*}$ theories with signature $1+9$ (one space and 9 time) together with theories in $0+10,2+8,3+7$ and $4+6$ dimensions. The supergravity theory in signature $(s, t)$ and its mirror with signature $(t, s)$ are equivalent, as will be discussed in section 11 . The $I I A_{5+5}$ and the $I I A_{5+5}^{*}$ actions are equivalent, related by taking $g_{\mu \nu} \rightarrow-g_{\mu \nu}$, and changing the overall sign of the action, while such a mirror transformation takes the $I I B_{5+5}$ theory to itself, and the II $B_{5+5}^{*}$ theory to itself. A map through this maze of T-dual 10-dimensional theories is given in figure 5. Note that this includes the square of theories in 9+1 dimensions given in figure 1, its mirror in $1+9$ dimensions and the square of theories in $5+5$ dimensions given in figure 4 . Reflection about the central horizontal line on which the $I I B_{5+5}$ theories lie takes each theory to its mirror. 


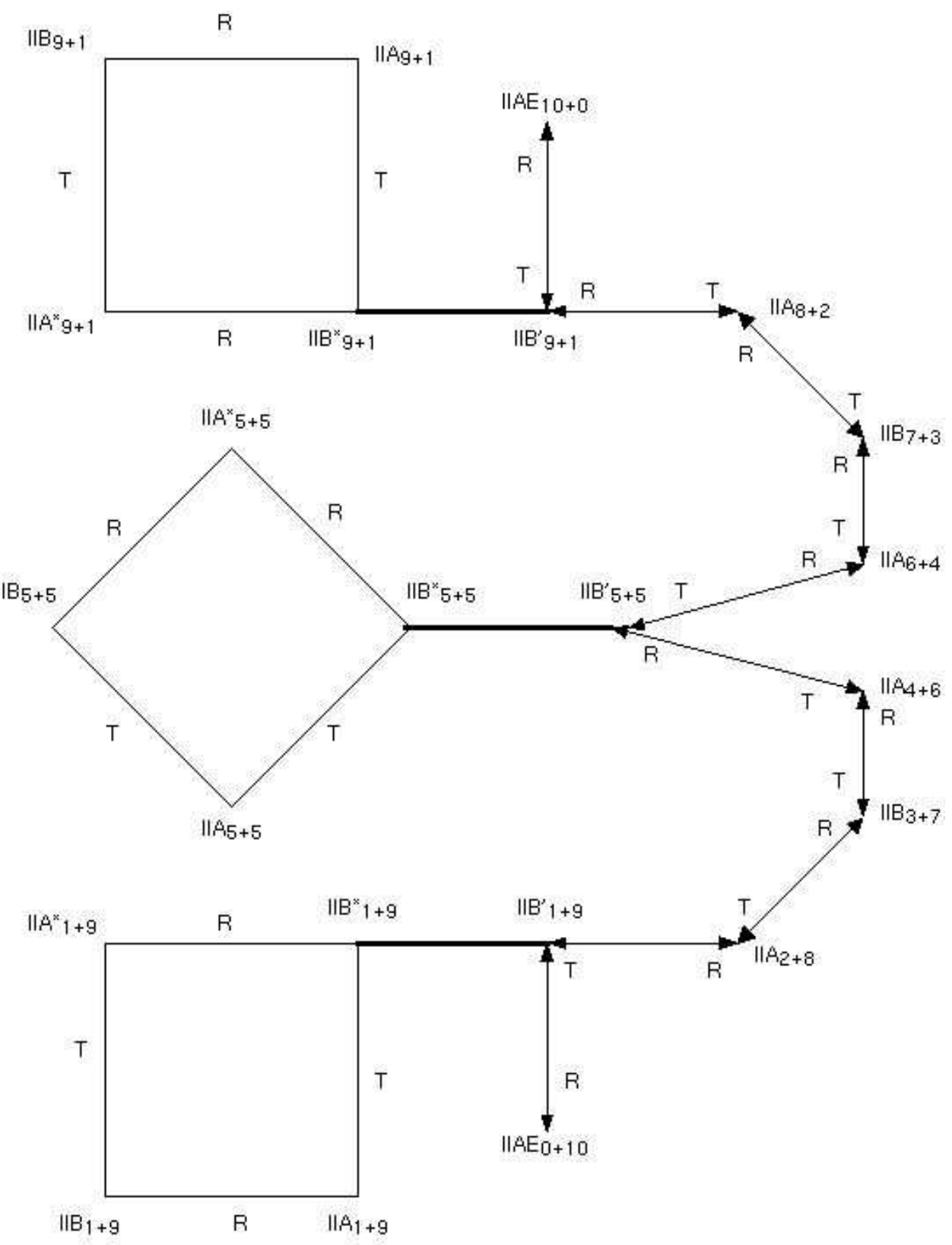

Figure 5 The dualities linking the type II string theories in 10 dimensions. A bold line represents an S-duality interchanging strong and weak coupling, a line with an $R$ indicates a spatial T-duality, a line with a $T$ indicates a timelike $\mathrm{T}$ duality, and a double-ended arrow with both an $R$ and a $T$ indicates a signature changing T-duality, where the theory at the $R$ end compactified on a spacelike circle of radius $R$ is T-dual to the theory at the $T$ end on a timelike circle of radius $T=1 / R$. 
The $I I A_{5+5}$ and $I I B_{5+5}$ theories have actions in which all of the kinetic terms have the 'right sign', so that the corresponding actions are the same as those for the usual $I I A$ and $I I B$ theories (4.1),(4.2), but with different signature. In $5+5$ dimensions there are Majorana-Weyl spinors as well as real self-dual 5-forms, just as in $9+1$ dimensions. The $I I A_{5+5}^{*}$ and $I I B_{5+5}^{*}$ string theories are obtained from the $I I A_{5+5}$ and $I I B_{5+5}$ string theories in the same way as the corresponding theories in $9+1$ dimensions, by acting with $i^{F_{L}}$, so that the signs of the RR gauge field kinetic terms are reversed so that the supergravity actions are of the form $(4.3),(4.4)$. The $I I A_{5+5}$ and $I I B_{5+5}$ theories have untwisted superalgebras

$$
\left\{Q_{i}, Q_{j}\right\}=\left(\Gamma^{\mu} C^{-1}\right) P_{\mu} \delta_{i j}
$$

while the $I I A_{5+5}^{*}$ and $I I B_{5+5}^{*}$ theories have the twisted superalgebra (2.1). The IIA and IIB string theories in signatures $9+1,5+5$, and the $I I A^{*}$ and $I I B^{*}$ string theories in signature $1+9$ are the only string theories in which all signs and phases are the conventional ones.

\section{The Strong-Coupling Limit of the Type $I I A^{*}$ Theory}

In this section, we will investigate the strong-coupling limit of the type $I I A^{*}$ theory. For the IIA theory, there are D0-branes with mass $m \sim n / g$ for all integers $n$ where $g$ is the string coupling, and these become light at strong coupling and are identified with a Kaluza-Klein tower of states from the decompactification of an extra spatial dimension [26]. For the $I I A^{*}$ theory, there are E1-branes with tachyonic mass $m, m^{2}=-\mu^{2}$ with $\mu$ real and $\mu \sim n / g$ for all integers $n$. Then $\mu \rightarrow 0$ at strong coupling and there is again an infinite tower of states that become massless in the limit and which fit into supergravity multiplets. Whereas the compactification of a spatial dimension gives a Kaluza-Klein tower of massive states, the compactification of a timelike dimension gives a Kaluza-Klein tower of tachyonic states, and in either case the tower becomes light as the extra dimension decompactifies. Thus it is natural to identify the strong coupling limit of the $I I A^{*}$ 
theory as one in which an extra timelike dimension decompactifies to give a theory in $9+2$ dimensions.

This result can be checked in a number of ways. It is certainly to be expected that the strong coupling limit should lead to the decompactification of an 11'th dimension, and the field-theory limit of the 11-dimensional theory should, on dimensional reduction, give the $I I A^{*}$ supergravity (4.3) in $9+1$ dimensions. This 11-dimensional field theory cannot be the usual 11-dimensional supergravity [27] with bosonic action

$$
S_{M}=\int d^{11} x \sqrt{-g}\left[R-G_{4}^{2}\right]+\frac{4}{3} \int G_{4} \wedge G_{4} \wedge C_{3}
$$

since the spatial reduction of this gives the $I I A$ theory, not the $I I A^{*}$ theory, while the timelike reduction gives a Euclidean theory, not one in $9+1$ dimensions. As the $I I A^{*}$ theory has a kinetic term for $C_{3}$ of the wrong sign, a natural guess would be a $10+1$ dimensional field theory of the form (7.1) but with the sign of the kinetic term of $G_{4}$ reversed. However, the spatial reduction of such an action would give kinetic terms for $B_{2}$ and $C_{1}$ differing in sign from those of the $I I A^{*}$ theory (4.3). However, the fact that the kinetic term of $C_{1}$ in (4.3) has the wrong sign means that if it is to be interpreted as the graviphoton from the reduction of an 11-dimensional metric, then the reduction must be on a timelike circle, not a space-like one. Consider, then, the following action in 9+2 dimensions:

$$
S_{M}=\int d^{11} x \sqrt{g}\left[R+G_{4}^{2}\right]+\frac{4}{3} \int G_{4} \wedge G_{4} \wedge C_{3}
$$

Its dimensional reduction on a timelike circle indeed gives the $I I A^{*}$ action, and if there is any 11-dimensional supergravity theory whose dimensional reduction gives the $I I A^{*}$ theory, it must have this bosonic sector. The strong coupling limit of the $I I A^{*}$ theory is then a theory in $9+2$ dimensions which we will denote as $M^{*}$ theory. The effective supergravity theory is given by coupling (7.2) to a gravitino which, as will be seen in section 11, is a pseudo-Majorana spinor (with 32 real components). 
Another way of obtaining the same theory is as follows. Consider first Mtheory compactified on a space-like 3 -torus, $T^{3}$. In the limit that the torus shrinks to zero size, one recovers M-theory [28]; dimensional reduction gives a theory in $7+1$ dimensions, but the modes from membranes wrapping around each of the three 2-cycles become light as the cycles shrink, and these light modes signal the opening up of three new spatial dimensions. If instead M-theory is compactified on a Lorentzian 3-torus, $T^{2,1}$, then in the limit that the torus shrinks to zero size, one again expects 3 new dimensions to open up, in addition to the 8 Euclidean dimensions from the dimensional reduction. However, in this case there is one Euclidean $T^{2}$ 2-cycle and two Lorentzian $T^{1,1} 2$-cycles, and we shall see that the shrinking Euclidean 2-cycle gives an extra space dimension while each shrinking Lorentzian 2-cycle gives an extra time dimension, resulting in a theory in $9+2$ dimensions.

M-theory on a Euclidean $T^{2}$ shrinking to zero size gives $8+1$ dimensions, as in the field theory limit, together with an extra spatial dimension from membranes wrapped on $T^{2}$, to give the IIB string in $9+1$ dimensions. On the other hand, as we have seen, M-theory on a Lorentzian $T^{1,1}$ shrinking to zero size gives 9 Euclidean dimensions, as in the field theory limit, together with an extra timelike dimension from membranes wrapped on $T^{1,1}$, to give the $I I B^{*}$ theory in $9+1$ dimensions. Thus membranes wrapped on a shrinking $T^{2,0}$ give an extra space dimension while those wrapped on a shrinking $T^{1,1}$ give an extra time dimension. This also follows from the fact that T-duality of Euclidean strings changes the signature. Then for M-theory on a shrinking $T^{2,1}$, one spatial and two timelike dimensions open up, to give $M^{*}$ theory in $9+2$ dimensions. M-theory and $M^{*}$ theory are then linked by a duality, and so are not independent theories: M-theory on a Lorentzian torus $T^{2,1}$ is equivalent to $M^{*}$-theory compactified on a dual torus which, as we will see in section 10 , is a torus $T^{1,2}$ with the opposite signature. 


\section{Compactifications of $M^{*}$ Theory}

Consider now reductions of $M^{*}$ theory, analogous to the ones considered above for M-theory. Reduction on a timelike circle gives the $I I A^{*}$ theory in $9+1$ dimensions, with coupling constant related to the radius so that the strong coupling limit of the $I I A^{*}$ theory is a decompactification to $M^{*}$ theory in $9+2$ dimensions. On the other hand, reduction on a spacelike circle gives a string theory in $8+2$ dimensions, which is the $I I A_{8+2}$ string theory obtained in section 6 . Considering toroidal reductions of $M^{*}$ theory clarifies some of the dualities represented in figure 5 .

First, reduction of $M^{*}$ theory on a torus $T^{0,2}$ with 2 timelike circles gives the moduli space in figure 6 .

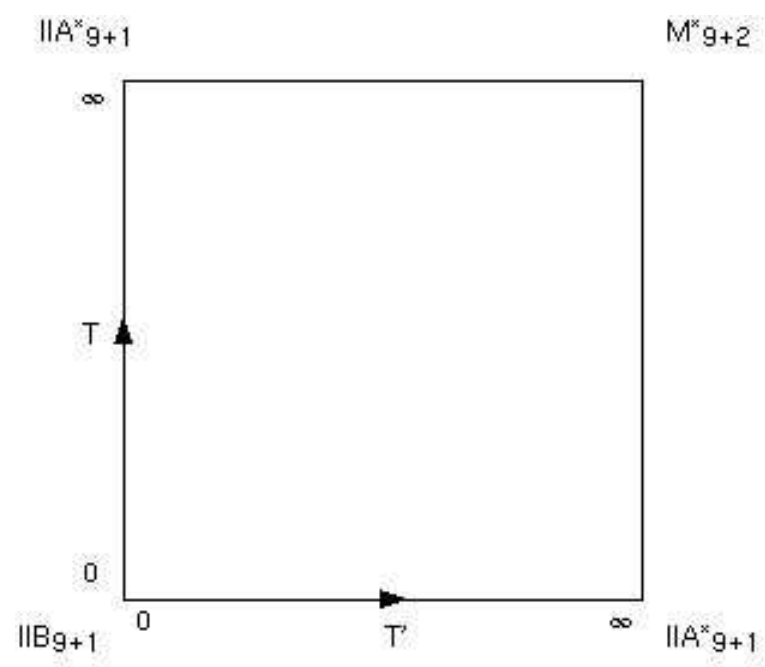

Figure 6 The moduli space for the compactification of $M^{*}$-theory from $9+2$ dimensions on a torus $T^{0,2}$ with two timelike circles of radii $T, T^{\prime}$. The figure should be identified under reflection in the $T=T^{\prime}$ diagonal.

The II $A^{*}$ and IIB theories are related by timelike T-duality and the limit as the $T^{0,2}$ shrinks to zero size gives the IIB theory.

Reduction of $M^{*}$ theory on a Lorentzian torus $T^{1,1}$ gives the following diagram: 


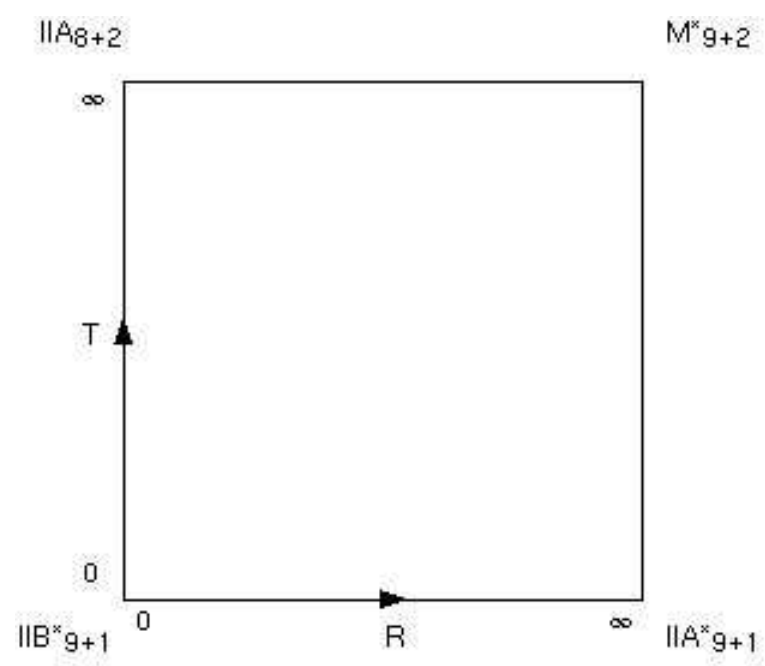

Figure 7 The moduli space for the compactification of $M^{*}$-theory from $9+2$ dimensions on a Lorentzian torus $T^{1,1}$ with spacelike radius $R$ and timelike radius $T$.

Compactification first on time leads to the T-duality between the $I I A^{*}$ theory on a spatial circle of radius $R$ and the $I I B^{*}$ theory on a spatial circle of radius $1 / R$. From section 4 , the $I I B^{\prime}$ string theory is the strong coupling limit of the $I I B^{*}$ string theory, and the $I I B^{\prime}$ and $I I A_{8+2}$ theories both have Euclidean fundamental strings. There is a signature-changing T-duality between the $I I A_{8+2}$ theory on a timelike circle of radius $T$ and the $I I B^{\prime}$ string theory on a spatial circle of radius $R=1 / T$, and this arises in fig. 5 from compactifying first on space. In the limit in which the $T^{1,1}$ shrinks to zero size, we obtain the $I I B^{*}$ theory with coupling constant $g \sim R / T$. The strong coupling limit of the $I I A_{8+2}$ theory is the $M^{*}$ theory, with a spacelike circle decompactifying.

Compactifying the $I I A_{8+2}$ theory on a spacelike circle of radius $R$ is equivalent to compactifying a T-dual theory on a timelike circle of radius $1 / R$, and the $\mathrm{T}$-dual theory must be in signature $7+3$, and we will denote it as $I I B_{7+3}$.

The moduli space for reduction of $M^{*}$ theory on a spatial torus $T^{2,0}$ is shown in figure 8 . The $M^{*}$ theory picture leads to the signature-changing T-duality between 
the $I I B_{7+3}$ theory and the $I I A_{8+2}$ theory, and the limit in which the $T^{2,0}$ shrinks to zero size gives the $I I B_{7+3}$ theory.

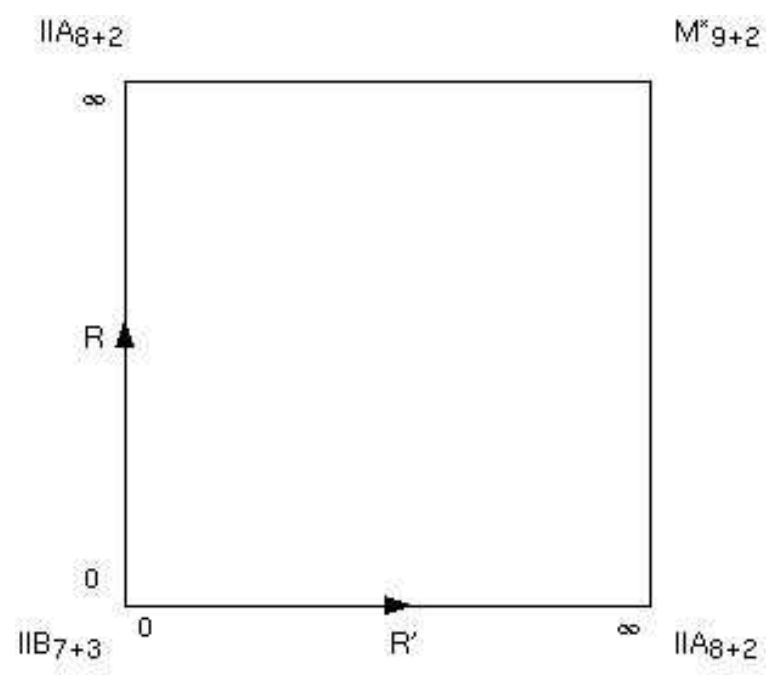

Figure 8 The moduli space for the compactification of $M^{*}$-theory from $9+2$ dimensions on a Euclidean torus $T^{2,0}$ with two spacelike circles of radii $R, R^{\prime}$. The figure should be identified under reflection in the $R+R^{\prime}$ diagonal.

\section{More Strong Coupling Limits}

The $I I A_{5+5}$ string in $5+5$ dimensions is very similar to the usual $I I A$ string in $9+1$ dimensions. In particular, it has D0-branes that become light at strong coupling, signalling the decompactification of a new spacelike dimension, so that its strong coupling limit is a theory in signature $6+5$ dimensions which we will refer to as the $M_{6+5}^{\prime}$ theory. This is also the strong coupling limit of the $I I A_{6+4}$ theory, which has E1-branes that become light at strong couling, signalling an extra time dimension. A supergravity theory in $6+5$ dimensions with lagrangian $R-\left(d C_{3}\right)^{2}+\ldots$ is the unique 11 dimensional theory that can be dimensionally reduced to give the $I I A_{5+5}$ supergravity (by reducing on a spacelike circle) or the $I I A_{6+4}$ supergravity (by reducing on a timelike circle). Similarly, the strongcoupling limit of the $I I A_{5+5}^{*}$ string and the $I I A_{4+6}$ string is a reversed-signature 
$M_{5+6}^{\prime}$ theory in $5+6$ dimensions. The strong coupling limits of the $I I A_{2+8}$ string and the $I I A_{1+9}^{*}$ strings are a reversed signature $M_{2+9}^{*}$ theory in $2+9$ dimensions and the strong coupling limits of the $I I A_{0+10}$ string and the $I I A_{1+9}$ strings are a reversed signature $M_{1+10}$ theory in $1+10$ dimensions.

Consider now the compactification of the $M^{\prime}$ theory on circles and 2-tori of various signatures. The reduction on a timelike circle gives the $I I A_{6+4}$ theory, while on a spacelike circle gives a $I I A_{5+5}$ theory in $5+5$ dimensions, which is $\mathrm{T}$ dual to a $I I B_{5+5}$ theory in $5+5$ dimensions on a spacelike circle. The moduli spaces for the compactifications on the tori $T^{2,0}, T^{1,1}$ and $T^{0,2}$ are shown in figures 9,10 and 11.

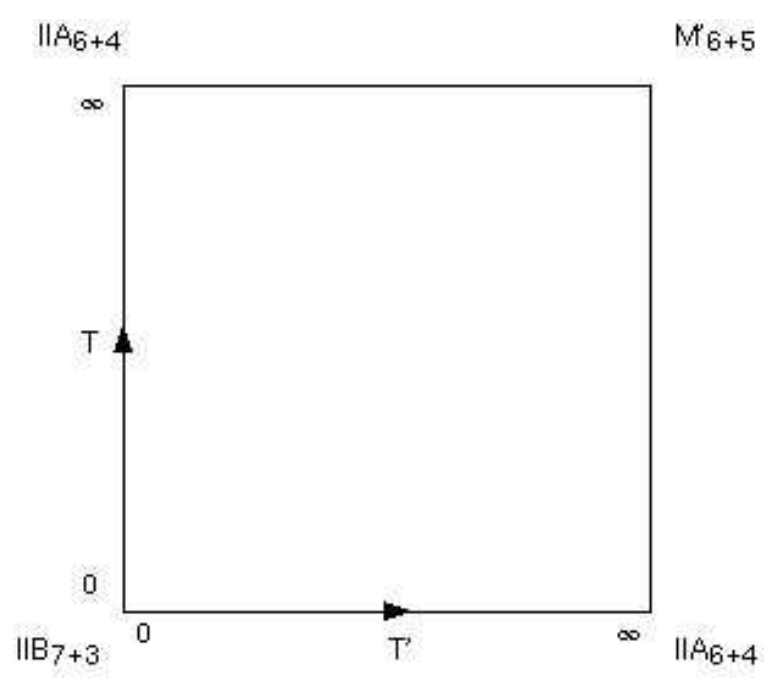

Figure 9 The moduli space for the compactification of $M^{\prime}$-theory from $6+5$ dimensions on a torus $T^{0,2}$ with two timelike circles of radii $T, T^{\prime}$. The figure should be identified under reflection in the $T=T^{\prime}$ diagonal. 


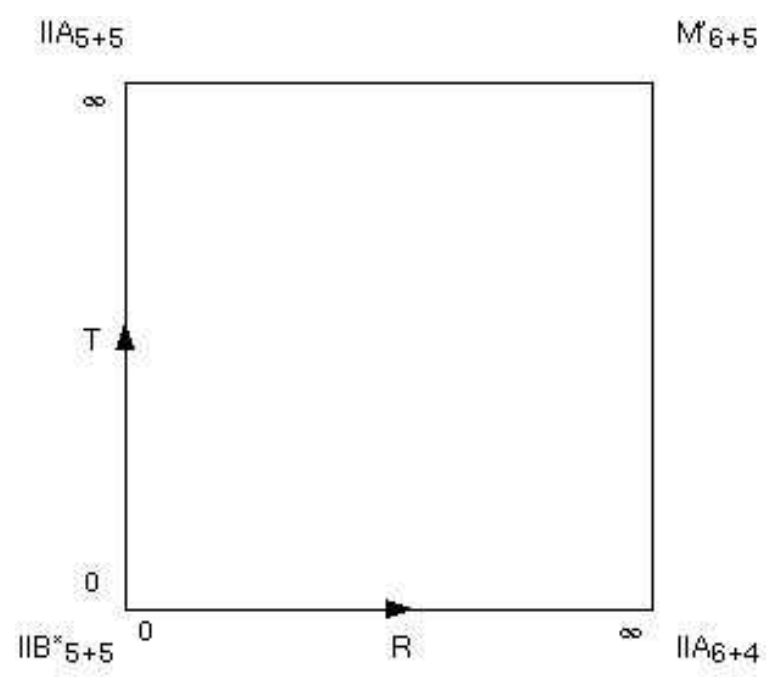

Figure 10 The moduli space for the compactification of $M^{\prime}$-theory from $6+5$ dimensions on a Lorentzian torus $T^{1,1}$ with spacelike radius $R$ and timelike radius $T$.

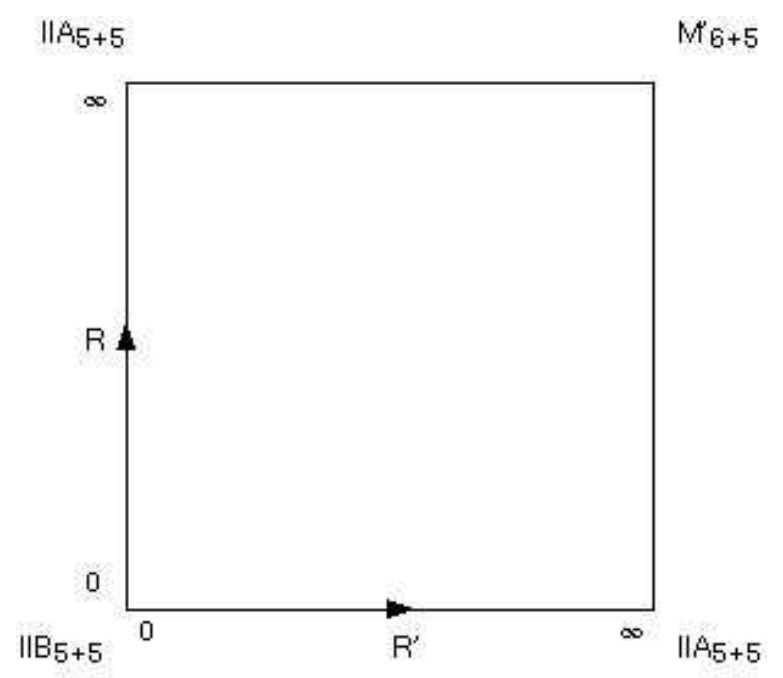

Figure 11 The moduli space for the compactification of $M^{\prime}$-theory from $6+5$ dimensions on a Euclidean torus $T^{2,0}$ with two spacelike circles of radii $R, R^{\prime}$. The figure should be identified under reflection in the $R=R^{\prime}$ diagonal.

In the limits in which the torus $T^{2,0}, T^{1,1}$ or $T^{0,2}$ shrinks to zero size, we get the $I I B_{5+5}, I I B_{5+5}^{*}$ or $I I B_{7+3}$ theories, respectively. 
To understand the remaining $5+5$ dimensional T-dualities in figure 4 from 11 dimensions, one needs to consider toroidal compactifications of the mirror theory in $5+6$ dimensions, which is the strong coupling limit of the type $I I A_{5+5}^{*}$ theory. These are depicted in figures $12,13,14$.

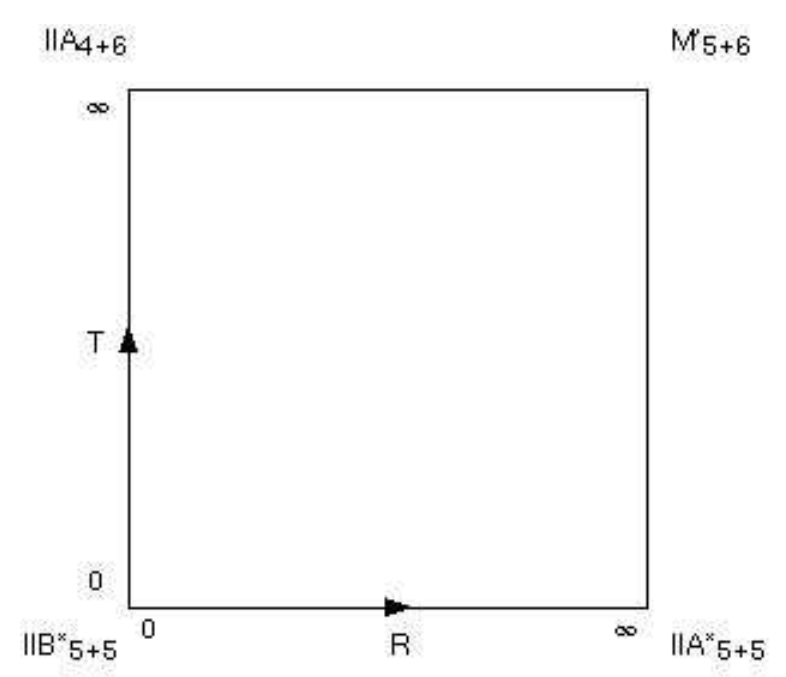

Figure 12 The moduli space for the compactification of $M^{\prime}$-theory from $5+6$ dimensions on a Lorentzian torus $T^{1,1}$ with spacelike radius $R$ and timelike radius $T$.

Figure 12 gives an $M^{\prime}$-theory understanding of the spacelike T-duality between the $I I A_{5+5}^{*}$ theory and the $I I B_{5+5}^{*}$ theory, while figure 13 leads to the timelike Tduality between the $I I A^{*}+{ }_{5+5}$ theory and the $I I B+{ }_{5+5}$ theory. Figure 14 leads to the signature-changing T-dualities between the $I I B_{3+7}$ and $I I A_{4+6}$ theories. 


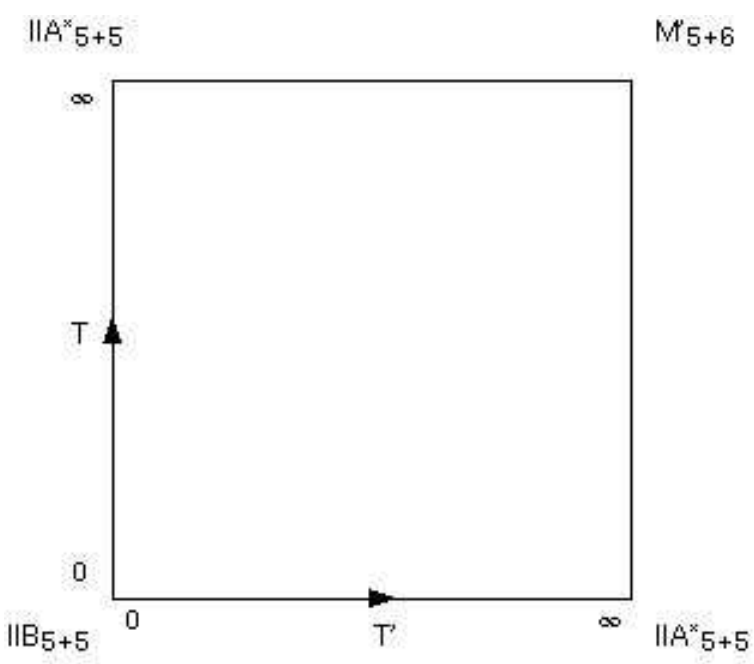

Figure 13 The moduli space for the compactification of $M^{\prime}$-theory from $5+6$ dimensions on a torus $T^{0,2}$ with two timelike circles of radii $T, T^{\prime}$. The figure should be identified under reflection in the $T=T^{\prime}$ diagonal.

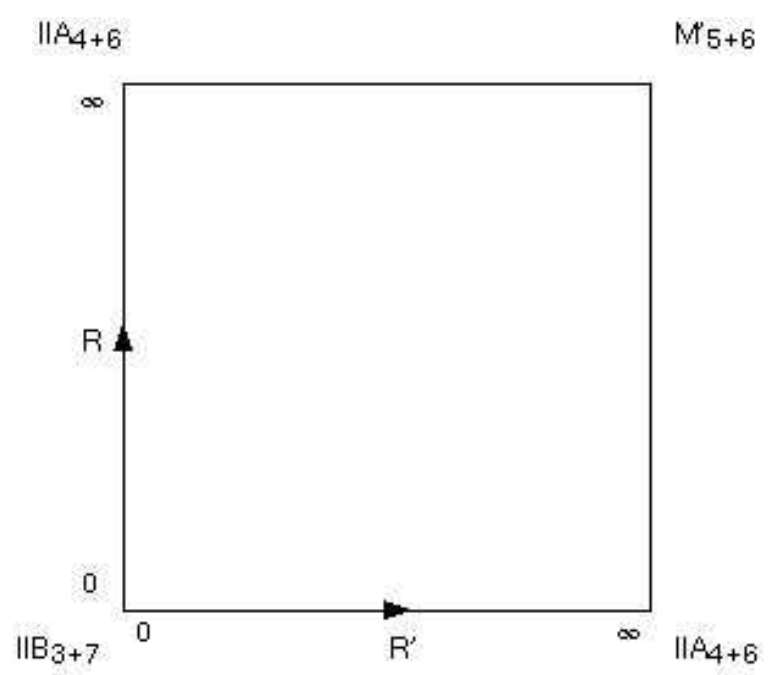

Figure 14 The moduli space for the compactification of $M^{\prime}$-theory from $5+6$ dimensions on a Euclidean torus $T^{2,0}$ with two spacelike circles of radii $R, R^{\prime}$. The figure should be identified under reflection in the $R=R^{\prime}$ diagonal. 


\section{Compactification on 3-Tori}

In this section we will consider the M-theories of various signatures compactified on 3-tori of various signatures, giving dualities linking the M-theories. Mtheory in $10+1$ dimensions compactified on $T^{2,0}$ gives the IIB string in the limit in which the torus shrinks, while when compactified on a shrinking Lorentzian torus $T^{1,1}$ it gives the $I I B^{*}$ string, as we have seen, so that a shrinking $T^{2,0}$ gives an extra spatial coordinate (in addition to the $8+1$ dimensions remaining after compactification), while a shrinking $T^{1,1}$ gives an extra time dimension (in addition to the 9+0 dimensions remaining after compactification). The extra dimension opens up because an infinite tower of states from the membrane wrapping modes contribute to the massless spectrum in the limit, and these can be associated with the KaluzaKlein modes from an extra dimension that is decompactifying. Then M-theory on $T^{3,0}$ gives 3 extra spatial dimensions in the shrinking limit from membranes wrapping around the three 2-cycles, so that these replace the three spatial dimensions lost in the compactification and a T-dual M-theory in $10+1$ non-compact dimensions is regained in the limit. On $T^{2,1}$, there are 2 Lorentzian $T^{1,1}$ cycles and one Euclidean $T^{2,0}$ cycle and so, in the limit in which all three circles shrink, the 2 spatial and one time dimensions that are lost are replaced by two time and one space dimensions, so that the $M^{*}$ theory in $9+2$ dimensions is regained.

Similar arguments can be applied to the $M^{*}$ and $M^{\prime}$ theories. From the duali-

ties in section 8 , we see that $M_{9+2}^{*}$ theory compactified on a shrinking $T^{2,0}$ gives an extra time dimension, on a shrinking $T^{1,1}$ gives an extra spatial dimension and on a shrinking $T^{0,2}$ gives an extra time dimension. Then $M_{9+2}^{*}$ theory compactified on a shrinking $T^{3,0}$ gives $6+2$ dimensions plus 3 extra time dimensions to give a theory in 6 space and 5 time dimensions, the $M_{6+5}^{\prime}$ theory. The $M_{9+2}^{*}$ theory compactified on a shrinking $T^{2,1}$ gives $7+1$ dimensions plus an extra two space and one time dimensions to give a theory in 9 space and 2 time dimensions, which is the $M_{9+2}^{*}$ theory again. The $M_{9+2}^{*}$ theory compactified on a shrinking $T^{1,2}$ gives $8+0$ dimensions plus an extra two space and one time dimensions to give a theory 
in 10 space and 1 time dimensions, which is the original $M_{10+1}$ theory.

From the dualities in section 9 , we see that $M_{6+5}^{\prime}$ theory compactified on a shrinking $T^{2,0}$ gives an extra spatial dimension, on a shrinking $T^{1,1}$ gives an extra time dimension and on a shrinking $T^{0,2}$ gives an extra spatial dimension. Then $M_{6+5}^{\prime}$ theory compactified on a shrinking $T^{3,0}$ gives $3+5$ dimensions plus 3 extra space dimensions to give a theory in 6 space and 5 time dimensions, the $M_{6+5}^{\prime}$ theory again. The $M_{6+5}^{\prime}$ theory compactified on a shrinking $T^{2,1}$ gives $4+4$ dimensions plus an extra two time and one space dimensions to give a theory in 5 space and 6 time dimensions, which is the $M_{5+6}^{\prime}$ theory. The $M_{6+5}^{\prime}$ theory compactified on a shrinking $T^{1,2}$ gives $5+3$ dimensions plus an extra two time and one space dimensions to give a theory in 6 space and 5 time dimensions, which is the $M_{6+5}^{\prime}$ theory. Finally, $M_{6+5}^{\prime}$ theory compactified on a shrinking $T^{0,3}$ gives $6+2$ dimensions plus 3 extra space dimensions to give a theory in 9 space and 2 time dimensions, the $M_{9+2}^{*}$ theory.

The torus reductions of the reversed signature cases are given by reversing all the signatures in the above. Then we have a chain of dualities linking the 'different' M-theories. $M_{10+1}$ theory on a shrinking $T^{2,1}$ gives the $M_{9+2}^{*}$ theory, the $M_{9+2}^{*}$ theory on a shrinking $T^{3,0}$ gives the $M_{6+5}^{\prime}$ theory, the $M_{6+5}^{\prime}$ on a shrinking $T^{2,1}$ gives the $M_{5+6}^{\prime}$ theory, the $M_{5+6}^{\prime}$ theory on a shrinking $T^{3,0}$ gives the $M_{2+9}^{*}$ theory and the $M_{2+9}^{*}$ theory on a shrinking $T^{2,1}$ gives the $M_{1+10}$ theory. The usual $10+1$ dimensional M-theory compactified on $T^{2,1}$ is equivalent to $M_{9+2}^{*}$ theory compactified on a dual $T^{1,2}, M_{9+2}^{*}$ theory compactified on $T^{3,0}$ is equivalent to $M_{6+5}^{\prime}$ theory compactified on a dual $T^{0,3}, M_{6+5}^{\prime}$ theory compactified on $T^{2,1}$ is equivalent to $M_{5+6}^{\prime}$ theory compactified on a dual $T^{1,2}$, and so on, where in each case, the dual torus has a reversed signature as well as the inverse volume. There are also self-dualities that preserve the signature, so that for example the $M_{9+2}^{*}$ theory compactified on $T^{2,1}$ is equivalent to the same theory compactified on a dual $T^{2,1}$. 


\section{Supersymmetry and Fermions}

The dualities linking the various theories obtained in previous sections guarantee that each of the theories has 32 supersymmetries, but it is interesting to check how the details work out. It will be useful to review the types of spinors that can occur for each spacetime signature [29,30], following [29]. In $D=s+t$ dimensions with $s$ space dimensions and $t$ time dimensions, the Clifford algebra is

$$
\left\{\Gamma^{m}, \Gamma^{n}\right\}=-2 \eta^{m n}
$$

where

$$
\eta^{m n}=\operatorname{diag}\left(-1_{t}, 1_{s}\right)
$$

and $m, n$ are tangent space indices. The gamma matrices can be chosen to satisfy

$$
\Gamma_{m}^{\dagger}=-(-1)^{t} A \Gamma_{m} A^{-1}
$$

where $A$ is the product of the timelike gamma matrices

$$
A=\Gamma_{1} \Gamma_{2} \ldots \Gamma_{t}
$$

For even $D, \pm \Gamma_{m}^{*}$ constitute an equivalent representation of the Clifford algebra so that there is a matrix $B$ such that

$$
\Gamma_{m}^{*}=\eta B \Gamma_{m} B^{-1}, \quad \eta= \pm 1
$$

for either choice of $\eta$, while for odd $D$ there is a matrix $B$ satisfying (11.5) only for

$$
\eta=(-1)^{(s+1-t) / 2}
$$

The matrix $B$ is unitary and satisfies

$$
B^{*} B=\epsilon(\eta, s, t), \quad \epsilon= \pm 1
$$

where $\epsilon$ depends on the choice of $\eta$ (if $D$ even) and on the signature $s, t$. The 
spacetime signatures $(s, t)$ allowing the four possible combinations $(\epsilon, \eta)$ are [29]

$$
\begin{aligned}
\epsilon=+1, \eta=-1: & s-t=0,1,2 \bmod 8 \\
\epsilon=+1, \eta=+1: & s-t=0,6,7 \bmod 8 \\
\epsilon=-1, \eta=-1: & s-t=4,5,6 \bmod 8 \\
\epsilon=-1, \eta=+1: & s-t=2,3,4 \bmod 8
\end{aligned}
$$

The charge conjugation matrix is

$$
C=B^{t} A
$$

and

$$
\Gamma_{m}^{t}=-(-1)^{t} \eta C \Gamma_{m} C^{-1}
$$

For even $D$, the matrix

$$
\Gamma_{D+1}=\Gamma_{1} \Gamma_{2} \ldots \Gamma_{D}
$$

satisfies

$$
\left(\Gamma_{D+1}\right)^{2}=(-1)^{(s-t) / 2}
$$

and the chiral projection operators

$$
P_{ \pm}=\frac{1}{2}\left(1 \pm(-1)^{(s-t) / 4} \Gamma_{D+1}\right)
$$

can be used to decompose a spinor into two Weyl spinors $\psi_{ \pm}=P_{ \pm} \psi$.

If $\epsilon=1$, then it is consistent to impose the reality constraint

$$
\psi^{*}=B \psi, \quad \epsilon=1
$$

on spinors. Spinors satisfying this are Majorana spinors if $\eta=-1$ and pseudoMajorana spinors if $\eta=1$. The two cases are distinct in general: for example, a 
Majorana fermion can be massive, but a pseudo-Majorana fermion cannot satisfy a massive Dirac equation and so must be massless [29]. The Dirac conjugate of a spinor $\psi$ is

$$
\bar{\psi}=\psi^{\dagger} A
$$

so a (pseudo-) Majorana spinor satisfies

$$
\bar{\psi}=\psi^{t} C
$$

If $\epsilon=-1$, the condition (11.14) is inconsistent as $B^{*} B=-1$, but if there are two spinors $\psi_{i}, i=1,2$, one can impose the condition

$$
\psi^{* i}=\left(\psi_{i}\right)^{*}=\varepsilon^{i j} B \psi_{j}
$$

where $\varepsilon^{i j}$ is the alternating tensor. Spinors satisfying this with $\eta=-1$ are symplectic Majorana spinors, while those satisfying this for $\eta=1$ are symplectic pseudo-Majorana spinors. Each of these four conditions is consistent with the chiral projection using (11.13) only if $s-t=0 \bmod 4$, in which case the resulting spinor is (symplectic) (pseudo) Majorana-Weyl.

The condition (11.14) can be generalised to the condition

$$
\psi^{* i}=\left(\psi_{i}\right)^{*}=M^{i j} B \psi_{j}, \quad \epsilon=1
$$

for $N$ spinors, $i=1, \ldots, j$, where $M^{i j}$ is a symmetric real matrix satisfying $M^{2}=1$, so that $M$ is invariant under $O(p, q)$ for some $p, q$ where $p+q=N$ is the number of spinors, and the condition (11.17) is invariant under the action of $O(p, q)$. We will refer to (11.18) as an $O(p, q)$-Majorana condition. If $\epsilon=-1$ and there are $2 N$ spinors, one can impose the condition

$$
\psi^{* i}=\left(\psi_{i}\right)^{*}=\Omega^{i j} B \psi_{j}
$$

for some antisymmetric real matrix $\Omega^{i j}$ satisfying $\Omega^{2}=-1$, and this is invariant under $\operatorname{Sp}(2 N)$. 
It is often possible to choose a Majorana representation of the gamma-matrices in which the gamma matrices are all real or all imaginary, so that one can take $B=1$ so that a (pseudo-) Majorana fermion is real, $\psi^{*}=\psi$. For example, in $9+1$ dimensions, the gamma matrices can be chosen to be all imaginary, $\Gamma_{m}^{*}=-\Gamma_{m}$, and there are two choices of $B$, depending on the sign of $\eta$. For $\eta=-1, \epsilon=1$, one can take $B=1$ so that a Majorana fermion is real, $\psi^{*}=\psi$. For $\eta=1, \epsilon=1$, one can instead choose $B=\Gamma_{11}$ and a pseudo-Majorana spinor satisfies $\psi^{*}=\Gamma_{11} \psi$. Then Majorana-Weyl spinors satisfy $\psi_{ \pm}^{*}=\psi_{ \pm}$while pseudoMajorana-Weyl spinors satisfy $\psi_{ \pm}^{*}= \pm \psi_{ \pm}$, so that a negative chirality pseudoMajorana-Weyl spinor is imaginary, while a positive chirality pseudo-MajoranaWeyl spinor is real. Then $\psi_{-} \rightarrow i \psi_{-}$takes a Majorana or Majorana-Weyl spinor to a pseudo-Majorana or pseudo-Majorana-Weyl spinor, and vice versa, so that Majorana and pseudo-Majorana conditions are equivalent in this sense, but the multiplication by $i$ changes the sign of the kinetic term of a $\psi_{-}$fermion. In particular, right-handed Majorana-Weyl and right-handed pseudo-Majorana-Weyl spinors are both real and so equivalent; in 10 dimensions, this applies to signatures $9+1,5+5,1+9$. Similarly, right-handed symplectic Majorana-Weyl spinors and right-handed symplectic pseudo-Majorana-Weyl spinors are equivalent; in 10 dimensions, this applies to signatures $7+3,3+7$. In signature $(1,9)$, all the gammamatrices can be chosen to be real (they are $i$ times the imaginary gamma matrices of the Majorana reperesentation in signature $9+1$ ) so that $B=1$ for $\eta=1$ and pseudo-Majorana spinors are real.

Given two Majorana-Weyl spinors $\psi^{ \pm}$of opposite chirality, they can be combined to form a Majorana spinor $\Psi=\left(\psi^{+}, \psi^{-}\right)$or a pseudo-Majorana spinor $\Psi=\left(\psi^{+}, i \psi^{-}\right)$. The IIA and $I I A^{*}$ supergravity theories in $9+1$ dimensions both have two gravitini $\psi_{\mu}^{+}, \psi_{\mu}^{-}$which are Majorana-Weyl spinors of opposite chirality. In the IIA supergravity, their kinetic terms have the same sign and can be written in terms of a Majorana gravitino as

$$
\int d^{10} x \sqrt{g} i \bar{\psi}_{\mu} \Gamma^{\mu \nu \rho} \nabla_{\nu} \psi_{\rho}
$$


or in terms of a pseudo-Majorana gravitino as

$$
\int d^{10} x \sqrt{g} i \bar{\psi}_{\mu} \Gamma^{\mu \nu \rho} \Gamma^{11} \nabla_{\nu} \psi_{\rho}
$$

In the $I I A^{*}$ supergravity, the kinetic terms of the two Majorana-Weyl gravitini have opposite sign and can be written in terms of a Majorana gravitino as (11.21) or in terms of a pseudo-Majorana gravitino as (11.20). In 10 dimensions, similar considerations apply to signatures $5+5,1+9$ in which there are also Majorana-Weyl spinors.

Two Majorana spinors $\psi, \chi$ can be combined into an $S O(2)$-Majorana spinor $\psi^{i}=(\psi, \chi), i=1,2$, satisfying (11.18) with $M^{i j}=\delta^{i j}$, or into an $S O(1,1)$ Majorana spinor $\psi^{i}=(\psi, i \chi)$ satisfying (11.18) with $M^{i j}=\eta^{i j}$, and similarly for pseudo-Majorana spinors or Majorana-Weyl spinors of the same chirality. The type IIB and type $I I B^{*}$ supergravities both have two Majorana-Weyl gravitini of the same chirality and the signs of their kinetic terms are the same for the type IIB supergravity and opposite for the type $I I B^{*}$ supergravity. In the type IIB theory, the gravitini can be combined into an $S O(2)$-Majorana-Weyl gravitino $\psi_{\mu}^{i}$ with kinetic term

$$
\int d^{10} x \sqrt{g} i \delta_{i j} \bar{\psi}_{\mu}^{i} \Gamma^{\mu \nu \rho} \nabla_{\nu} \psi_{\rho}^{j}
$$

or into an $S O(1,1)$-Majorana-Weyl gravitino $\psi_{\mu}^{i}$ with kinetic term

$$
\int d^{10} x \sqrt{g} i \eta_{i j} \bar{\psi}_{\mu}^{i} \Gamma^{\mu \nu \rho} \nabla_{\nu} \psi_{\rho}^{j}
$$

The two Majorana-Weyl gravitini of the type $I I B_{9+1}^{*}$ theory can be combined into either an $S O(2)$-Majorana-Weyl gravitino $\psi_{\mu}^{i}$ with kinetic term (11.23) or into an $S O(1,1)$-Majorana-Weyl gravitino $\psi_{\mu}^{i}$ with kinetic term (11.22).

For every theory in signature $(s, t)$ we have found a mirror theory with signature $(t, s)$. The bosonic part of the supergravity actions of the mirror pairs are equivalent (up to an overall sign), but there appear to be differences in some cases in the 
fermionic sectors. For example, there are Majorana fermions in signature $10+1$ but not in signature $1+10$, where, from above, only pseudo-Majorana fermions are allowed. In 11 dimensions, the fermions are Majorana in signatures $(10,1),(6,5)$ and $(2,9)$ and are pseudo-Majorana in the mirror signatures $(1,10),(5,6)$ and $(9,2)$, and in each case the spinors have 32 real components. However, a Majorana spinor in $10+1$ and a pseudo-Majorana spinor in $1+10$ dimensions are both real in a Majorana representation, so that the two mirror theories are equivalent, and similarly for other mirror pairs.

Indeed, consider a theory in signature $(s, t)$ with the spinors satisfying a Majorana-type condtion $\psi^{*}=N B \psi$ (which can be pseudo or symplectic) for a $B$-matrix with corresponding $\epsilon, \eta$, and for some matrix $N$ which is the identity for (pseudo)-Majorana spinors and is antisymmetric $N=\Omega$ for symplectic (pseudo)Majorana spinors. Taking $g_{\mu \nu} \rightarrow-g_{\mu \nu}, \Gamma_{\mu} \rightarrow i \Gamma_{\mu}$, gives a theory in signature $(t, s)$ in which the spinors still satisfy $\psi^{*}=N B \psi$, but $\eta$ has changed sign, so that a (symplectic) Majorana spinor changes to (symplectic) pseudo-Majorana, or vice versa. This mirror theory will also be supersymmetric, and so must be the same as the mirror obtained via the chain of dualities, as supersymmetry specifies the theory uniquely. Thus the mirror pairs of theories are all equivalent.

Dimensionally reducing to 10 dimensions on either a spacelike or a timelike circle, we obtain IIA theories in which the spinor is a 32-component Majorana or pseudo-Majorana spinor, depending on the signature as follows:

$$
\begin{aligned}
\text { Majorana : } & \text { II } A_{9+1}, I I A_{10+0}, I I A_{6+4}, I I A_{5+5}, I I A_{2+8}, I I A_{1+9}^{*} \\
\text { Pseudo-Majorana : } & I I A_{9+1}^{*}, I I A_{8+2}, I I A_{5+5}^{*}, I I A_{4+6}, I I A_{1+9}, I I A_{0+10}
\end{aligned}
$$

In signatures $9+1,5+5,1+9$, the spinors can be decomposed into Majorana-Weyl spinors. Note that two kinds of spinors are possible for each signature (e.g. $9+1$ dimensions allows Majorana or pseudo-Majorana spinors), but the relation with 11 dimensions picks out one type in each case. However, in signatures $(9,1),(1,9)$ and $(5,5)$, the pseudo-Majorana spinors can be repackaged as Majorana spinors, as seen above. 
The 11-dimensional superalgebra for all of the signatures is of the form

$$
\{Q, Q\}=\left(\Gamma_{M} C^{-1}\right) P^{M}
$$

where $C$ is the $D=11$ charge conjugation matrix and the supercharges $Q$ are either Majorana or pseudo-Majorana, depending on the signature. A spatial dimensional reduction will give a superalgebra of the same form in $D=10$, while for a reduction on a timelike direction $x^{11}$, the 10-dimensional charge conjugation matrix $C_{10}$ is related to that in 11-dimensions by $C=C_{10} \Gamma_{11}$, so that the 10-dimensional algebra is of the twisted form

$$
\{Q, Q\}=\left(\Gamma_{M} \Gamma_{11} C_{10}^{-1}\right) P^{M}
$$

For signatures $(9,1),(5,5)$ and $(1,9)$ this can be rewritten in terms of two (pseudo)Majorana-Weyl supercharges $Q_{i}, i=1,2$, of opposite chirality, in which case it becomes

$$
\left\{Q_{i}, Q_{j}\right\}=\eta_{i j}\left(\Gamma_{M} C^{-1}\right) P^{M}
$$

where $\eta_{i j}=\operatorname{diag}(+1,-1)$, so that $(2.1)$ is recovered. This is a general feature: each timelike reduction introduces a twist into the superalgebra. In $9+1,5+5$ or $1+9$ dimensions, the Majorana-Weyl supercharges $Q_{i}$ can be combined into a Majorana or a pseudo-Majorana supercharge, and the twisted superalgebra (11.27) can be written as (11.26) in terms of a Majorana supercharge or as (11.25) in terms of a pseudo-Majorana supercharge.

For the IIB theories in signatures $9+1,5+5,1+9$, there are two Majorana-Weyl supercharges $Q_{i}$ of the same chirality which satisfy an untwisted superalgebra (6.1) for the $I I B_{9+1}, I I B_{5+5}, I I B_{1+9}$ theories, or a twisted superalgebra for the II $B_{9+1}^{*}, I I B_{5+5}^{*}, I I B_{1+9}^{*}$ theories. (These can be rewritten in terms of either an $S O(2)$-Majorana-Weyl supercharge or an $S O(1,1)$-Majorana-Weyl supercharge.) For the $I I B_{7+3}$ theory and the the $I I B_{3+7}$ the supercharges $Q_{i}, i=1,2$, form a 
symplectic Majorana-Weyl spinor, satisfying (11.17), and the superalgebra takes the symplectic twisted form

$$
\left\{Q_{i}, Q_{j}\right\}=\epsilon_{i j}\left(\Gamma_{M} C^{-1}\right) P^{M}
$$

\section{The Set of Theories}

We have found versions of M-theory with signatures $(10,1),(9,2),(6,5)$ and the mirrors $(1,10),(2,9),(5,6)$, and these are all linked by dualities - we saw in section 10 that we could get from one to another by compactifying on a 3-torus of suitable signature and taking the limit in which it shrinks to zero size. All can be linked by dualities in this way, so that they should not be regarded as different theories, but as different limits of the same underlying theory. All have field theory limits which are 11-dimensional supergravity theories with various signatures. Compactifying on a spacelike circle or a timelike circle gives IIA string theories with signatures $(10,0),(9,1),(8,2),(6,4),(5,5)$ together with their mirrors, and the starred versions of the theories with signature $(9,1),(1,9)$ and $(5,5)$ in which the signs of the RR fields are reversed. Compactifying the various M-theories on shrinking 2-tori $T^{2,0}, T^{1,1}$ or $T^{0,2}$ give IIB theories in signatures $(9,1),(7,3),(5,5)$ together with their mirrors, and the starred versions of the theories with signature $(9,1),(1,9)$ and $(5,5)$. The strong coupling limit of the $I I B^{*}$ theories gives the $I I B^{\prime}$ theories, while the remaining IIB theories are self-dual, so that their strong coupling limit is isomorphic to the weak coupling limit, as in the usual IIB theory [31]. The various 10-dimensional string theories are all linked by T-dualities or by S-dualities (taking weak coupling to strong coupling), as shown in figure 4.

The various 11-dimensional supergravities have a field content of a metric, a 3form gauge field and a gravitino which is Majorana for signatures $10+1,6+5,2+9$ and pseudo-Majorana for the mirror signatures $1+10,5+6,9+2$. The bosonic Lagrangian is of the form $R-\left(d C_{3}\right)^{2}+\ldots$ for signatures $10+1,6+5,2+9$ 
while the 3-form gauge field kinetic term has the wrong sign for the signatures $1+10,5+6,9+2$, with lagrangian $R+\left(d C_{3}\right)^{2}+\ldots$

The bosonic fields of the type IIA supergravities are the NS-NS fields $g_{\mu \nu}, B_{2}, \Phi$ and the RR gauge fields $C_{1}, C_{3}$ with a bosonic action of the form

$$
\begin{aligned}
S_{I I A}=\int d^{10} x & \sqrt{|g|}\left[e^{-2 \Phi}\left(R+4(\partial \Phi)^{2}\right)\right. \\
& \left.-\left( \pm e^{-2 \Phi} H^{2} \pm G_{2}^{2} \pm G_{4}^{2}\right)\right]+\ldots
\end{aligned}
$$

with the signs of the kinetic terms of $B_{2}, C_{1}, C_{3}$ for the various spacetime signatures given in table 1 (where a + sign is the usual sign, and - is the sign for a ghost-like field).

\begin{tabular}{|c|c|c|c|c|}
\hline Theory & $C_{1}$ Sign & $B_{2}$ Sign & $C_{3}$ Sign & Spinor \\
\hline$I I A_{9+1}$ & + & + & + & $\mathrm{M}=\mathrm{MW}+\mathrm{MW}$ \\
\hline$I I A_{9+1}^{*}$ & - & + & - & $\mathrm{PM}=\mathrm{MW}+\mathrm{MW}$ \\
\hline$I I A_{10+0}$ & - & - & + & $\mathrm{M}$ \\
\hline$I I A_{8+2}$ & + & - & - & $\mathrm{PM}$ \\
\hline$I I A_{6+4}$ & - & - & + & $\mathrm{M}$ \\
\hline$I I A_{5+5}$ & + & + & + & $\mathrm{M}=\mathrm{MW}+\mathrm{MW}$ \\
\hline$I I A_{5+5}^{*}$ & - & + & - & $\mathrm{PM}=\mathrm{MW}+\mathrm{MW}$ \\
\hline$I I A_{4+6}$ & + & - & - & $\mathrm{PM}$ \\
\hline$I I A_{2+8}$ & - & - & + & $\mathrm{M}$ \\
\hline$I I A_{1+9}^{*}$ & + & + & + & $\mathrm{M}=\mathrm{MW}+\mathrm{MW}$ \\
\hline$I I A_{1+9}$ & - & + & - & $\mathrm{PM}=\mathrm{MW}+\mathrm{MW}$ \\
\hline$I I A_{0+10}$ & + & - & - & $\mathrm{PM}$ \\
\hline
\end{tabular}

Table 1 The signs of the kinetic terms in (12.1) for the gauge fields $C_{1}, B_{2}, C_{3}$ for the $I I A_{s+t}$ theory in $s$ space and $t=10-s$ time dimensions. A ' + ' sign is the conventional sign. The spinors are Majorana (M) or pseudo-Majorana (PM), and in certain cases split into positive chirality Majorana-Weyl spinors (MW) and negative chirality ones (MW').

For the type IIB supergravities, the bosonic fields are the NS-NS fields $g_{\mu \nu}, B_{2}, \Phi$ and the RR fields $C_{0}, C_{2}, C_{4}$, where the $C_{4}$ field strength $G_{5}$ is self- 
dual, and the bosonic action is of the form

$$
\begin{aligned}
S_{I I B}=\int d^{10} x & \sqrt{|g|}\left[e^{-2 \Phi}\left(R+4(\partial \Phi)^{2}\right)\right. \\
& \left.-\left( \pm e^{-2 \Phi} H^{2} \pm G_{1}^{2} \pm G_{3}^{2} \pm G_{5}^{2}\right)\right]+\ldots
\end{aligned}
$$

with the signs of the kinetic terms of $B_{2}, C_{0}, C_{2}, C_{4}$ for the various spacetime signatures given in table 2 .

\begin{tabular}{|c|c|c|c|c|c|}
\hline Theory & $C_{0}$ Sign & $B_{2}$ Sign & $C_{2}$ Sign & $C_{4}$ Sign & Spinor \\
\hline$I I B_{9+1}$ & + & + & + & + & $\mathrm{MW}+\mathrm{MW}$ \\
\hline$I I B_{9+1}^{*}$ & - & + & - & - & $\mathrm{MW}+\mathrm{MW}$ \\
\hline$I I B_{9+1}^{\prime}$ & - & - & + & - & $\mathrm{MW}+\mathrm{MW}$ \\
\hline$I I B_{7+3}$ & + & - & - & + & $\mathrm{SMW}$ \\
\hline$I I B_{5+5}$ & + & + & + & + & $\mathrm{MW}+\mathrm{MW}$ \\
\hline$I I B_{5+5}^{*}$ & - & + & - & - & $\mathrm{MW}+\mathrm{MW}$ \\
\hline$I I B_{5+5}^{\prime}$ & - & - & + & - & $\mathrm{MW}+\mathrm{MW}$ \\
\hline$I I B_{3+7}$ & + & - & - & + & $\mathrm{SMW}$ \\
\hline$I I B_{1+9}$ & + & + & + & + & $\mathrm{MW}+\mathrm{MW}$ \\
\hline$I I B_{1+9}^{*}$ & - & + & - & - & $\mathrm{MW}+\mathrm{MW}$ \\
\hline$I I B_{1+9}^{\prime}$ & - & - & + & - & $\mathrm{MW}+\mathrm{MW}$ \\
\hline
\end{tabular}

Table 2 The signs of the kinetic terms in (12.2) for the gauge fields $C_{0}, B_{2}, C_{2}, C_{4}$ for the $I I B_{s+t}$ theory in $s$ space and $t=10-s$ time dimensions. A ' + ' sign is the conventional sign. The spinors are positive chirality Majorana-Weyl (MW) or symplectic Majorana-Weyl (SMW).

For the IIB theories, the scalar coset space is $S L(2, \mathbb{R}) / S O(2)$ when the $C_{0}$ sign is + and is $S L(2, \mathbb{R}) / S O(1,1)$ when the $C_{0} \operatorname{sign}$ is -.

Note that the theories are named so that the signature-reversing mirror transformation takes the $I I A_{9+1}$ theory to the $I I A_{1+9}$ theory, the $I I A_{9+1}^{*}$ theoryto the $I I A_{1+9}^{*}$ theory, the $I I B_{9+1}$ theory to the $I I B_{1+9}$ theory, the $I I B_{9+1}^{*}$ theory to the $I I B_{1+9}^{*}$ theory, and the $I I B_{9+1}^{\prime}$ theory to the $I I B_{1+9}^{\prime}$ theory. The mirror transformation also takes the $I I A_{5+5}$ theory to the $I I A_{5+5}^{*}$ theory, but leaves each of the three IIB theories in $5+5$ dimensions invariant. 
There are Majorana-Weyl spinors in signatures $9+1,1+9$ and $5+5$ and it is only in these signatures in which $N=1$ theories with 16 supersymmetries are possible, and it is in these signatures that supersymmetric Yang-Mills theories, type I strings or heterotic strings can occur. Orientifolding the type $I I B, I I B^{*}$ and $I I B^{\prime}$ theories in $9+1,5+5$ and $1+9$ dimensions gives type $I, I^{*}$ and $I^{\prime}$ theories in these signatures. The type I theories have both open strings and D-strings with Lorentzian world-sheets, the type $I^{*}$ theories have open strings with Lorentzian world-sheets and D-strings with Euclidean world-sheets, while the type $I^{\prime}$ strings have Euclidean open strings and Lorentzian D-strings. At strong coupling, the D-strings become, as in [32-34], the fundamental strings of the corresponding dual heterotic theories, which will be denoted $H O, H O^{*}, H O^{\prime}$ respectively. There are also $H E$-type heterotic strings in these signatures that can be obtained by compactifying $M, M^{*}, M^{\prime}$ theories on a spacelike or a timelike $S^{1} / \mathbb{Z}_{2}$, when possible. For example reducing M-theory on a spacelike $S^{1} / \mathbb{Z}_{2}$ gives the $H E_{9+1}$ theory, as in [35], reducing $M_{9+2}^{*}$ theory on a timelike $S^{1} / \mathbb{Z}_{2}$ gives a $H E_{9+1}^{*}$ theory with fundamental strings with Euclidean world-sheets, reducing $M_{6+5}^{\prime}$ theory on a spacelike $S^{1} / \mathbb{Z}_{2}$ gives the $H E_{5+5}$ theory with Lorentzian world-sheets, and reducing the $M_{5+6}^{\prime}$ theory on a timelike $S^{1} / \mathbb{Z}_{2}$ gives the $H E_{5+5}^{*}$ theory with Euclidean worldsheets. The various heterotic strings are then related to each other by T-dualities, and to type II theories by generalisations of the dualities in [31]. For example, the type $I I A_{5+5}$ theory compactified on $\mathrm{K} 3$ is dual to the $\mathrm{HO}_{5+5}$ or $\mathrm{HE}_{5+5}$ heterotic theory compactified on $T^{4}$, while the $I I A_{9+1}^{*}$ theory on $\mathrm{K} 3$ is dual to the $H O_{5+5}^{*}$ or $\mathrm{HE}_{5+5}^{*}$ heterotic theory on a timelike $T^{4}$. Such dualities can be used to relate heterotic timelike T-duality to type II timelike T-duality.

Compactifications of type IIB theory on a space $K$ for which the complex scalar field $\tau$ is not a function on $K$ but is the section of a bundle can be described as a compactification of F-theory on a $T^{2}$ bundle $B$ over $K$ [36]. If $K$ is an $n$ dimensional Euclidean space, then $B$ is an $n+2$-dimensional Euclidean space, and the F-theory background is $11+1$ dimensional. On the other hand, compactifications of type $I I B_{9+1}^{*}$ theory on a space $K$ for which the scalars taking values in 
$S L(2, \mathbb{R}) / S O(1,1)$ is the section of a bundle over $K$ can be described as a compactification of an $F^{*}$-theory on a $T^{1,1}$ bundle $B$ over $K$. If $K$ is an $n$-dimensional Euclidean space, then $B$ is an $n+2$-dimensional space with Lorentzian signature, and the $F^{*}$-theory background is $10+2$ dimensional, with two times [2]. Similarly, compactifications of the various $I I B$ theories in signatures $9+1,7+3,5+5$ and their mirrors in which the scalars have non-trivial transition functions can be described as compactifications of F-type theories in signatures $11+1,10+2,9+3,7+5,6+6$ and their mirrors.

Acknowledgements: I would like to thank Gary Gibbons, Ramzi Khuri and Greg Moore for helpful discussions.

\section{REFERENCES}

1. G. Moore, hep-th/9305139, 9308052 .

2. C.M. Hull, J.High Energy Phys. 7, (1998) 021; hep-th/9806146.

3. C.M. Hull and R.R. Khuri, hep-th/9808069.

4. J. Maldacena, hep-th/9711200.

5. E. Bergshoeff and E. Sezgin, Phys. Lett. B292 (1992) 87; S.V. Ketov, H. Nishino and S.J. Gates, Phys. Lett. B297 (1992) 99, Phys. Lett. B307 ( 1993) 323 and Nucl.Phys. B393 (1993) 149.

6. M.P. Blencowe and M.J. Duff, Nucl.Phys.B310:387,1988.

7. G.W. Gibbons and D.A. Rasheed, hep-th/904177.

8. A. Chamblin and R. Empran, hep-th/9607236.

9. P. Goddard, 'The Work of R.E. Borcherds', to appear in Proceedings of the International Congress of Mathematicians, and references therein.

10. J. Dai, R.G. Leigh and J. Polchinski, Mod. Phys. Lett. A4 (1989) 2073.

11. M. Dine, P. Huet and N. Seiberg, Nucl. Phys. B322 (1989) 301. 
12. C. M. Hull and B. Julia, hep-th/9803239.

13. E. Cremmer, I.V. Lavrinenko, H. Lu, C.N. Pope, K.S. Stelle and T.A. Tran, hep-th/9803259.

14. D. Friedan, E. Martinec and S. Shenker, Nucl. Phys. B271 (1986) 93.

15. S. Chaudhuri, C. Johnson, and J. Polchinski, "Notes on D-branes," hepth/9602052; J. Polchinski, "TASI Lectures on D-branes," hep-th/9611050; C.P. Bachas, hep-th/9806199.

16. P. Aspinwall, Nucl. Phys. Proc. Suppl. 46 (1996) 30, hep-th/9508154; J. H. Schwarz, hep-th/9508143.

17. C.M. Hull, Nucl.Phys. B468 (1996) 113 hep-th/9512181.

18. E. Bergshoeff, C.M. Hull and T. Ortin, Nucl. Phys. B451 (1995) 547, hep-th/9504081.

19. G.W. Gibbons, hep-th/9803206.

20. T. H. Buscher, Phys. Lett. 159B (1985) 127, Phys. Lett. B194 (1987), 51 ; Phys. Lett. B201 (1988), 466.

21. M. Roček and E. Verlinde, Nucl. Phys. B373 (1992), 630.

22. A. Giveon, M. Roček, Nucl. Phys. B380 (1992), 128.

23. E. Alvarez, L. Alvarez-Gaumé, J.L. Barbon and Y. Lozano, Nucl. Phys. B415 (1994) 71.

24. A. Giveon, M. Porrati and E. Rabinovici, Phys. Rep. 244 (1994) 77.

25. C.M. Hull and B. Spence, Phys. Lett. 232B (1989) 204.

26. E. Witten, Nucl. Phys. B443 (1995) 85, hep-th/9503124.

27. E. Cremmer and B. Julia, Phys. Lett. 80B (1978) 48; Nucl. Phys. B159 (1979) 141.

28. W. Fischler, E. Halyo, A. Rajaraman and L. Susskind, hep-th/9703102. 
29. T. Kugo and P.K. Townsend, Nucl. Phys. B221 (1983) 357.

30. P. van Nieuwenhuizen, Trieste lectures, 1981.

31. C.M. Hull and P.K. Townsend, hep-th/9410167.

32. C.M. Hull, Phys. Lett. B357 (1995) 545, hep-th/9506194.

33. A. Dabholkar, Phys. Lett. B357 (1995) 307, hep-th/9506160.

34. J. Polchinski and E. Witten, hep-th/9510169.

35. P. Horava and E. Witten, Nucl.Phys. B475 (1996) 94.

36. C. Vafa, Nucl. Phys. 469 (1996) 403. 